\title{
Principle of Link Evaluation
}

\author{
Jinbao ZHANG ${ }^{1}$, Hongming ZHENG ${ }^{2}$, Zhenhui TAN ${ }^{3}$, Yueyun CHEN ${ }^{4}$ \\ ${ }^{1,3}$ Institution of Electronics and Information, Beijing JiaoTong University, Beijing, China \\ ${ }^{2}$ Intel China Research Centre, Beijing, China \\ ${ }^{4}$ University of Science and Technology Beijing, Beijing, China \\ Email: ${ }^{1}$ jinbao.zhang@gmail.com, ${ }^{2}$ hongming.zheng@intel.com, ${ }^{3}$ zhhtan@bjtu.edu.cn, ${ }^{4}$ chenyy@ies.ustb.edu.cn
}

\begin{abstract}
Link Evaluation (LE) is proposed in system evaluation to reduce complexity. It is important to practical systems also for link adaptation. Current algorithms for link evaluation are developed by simulation method, lacking of theoretical description. Although they provide some good accuracy for some scenarios, all of them are not universal. With the help of information theory, a universal principle of link evaluation is proposed in this paper, which explains current algorithms and leads to a universal algorithm to implement link evaluation for common wireless transmissions.

This paper proposes an Extended Received Block Information Rate (ERBIR) algorithm for universal link evaluation, which is extended from current RBIR algorithm by the help of the principle presented in this paper. Mainly the universality and accuracy are highlighted. Simulation results verify all the algorithms mentioned in this paper. Both the principle and ERBIR are validated by simulation with various wireless scenarios.
\end{abstract}

Keywords: link evaluation, information theory, MIMO, OFDM, WiMAX II

\section{Introduction}

LINK evaluation aims to estimate the instant performance of transmissions for given channel status information (CSI), by a computational model with reasonable complexity.

As to wireless transmissions, due to macro and micro fading, the CSI is varying within both time and frequency domains, so fading will influence wireless transmission a lot. Consequently, link evaluation is significant to analysis and design for real wireless system.

For that the instant performance for wireless transmissions under given CSI can be computed by link evaluation quite simply and accurately, it is possible for System Level Simulation (SLS) to hold down real coding and decoding procedures, reducing a lot of complexity [1]. Meanwhile, wireless system can dynamically choose the proper transmitting mode with the help of link evaluation to enhance system performance, which is referred as link adaptation [1-3].

Accuracy is very important to link evaluation. For SLS, obviously it directly determines if the simulation results are reasonable. For link adaptation, accurate link evaluation ensures that the transmitting mode is selected properly. If the link performance is overestimated, the transmitter will always choose a mode which can not be supported by instantaneous CSI, introducing too much transmission error; while the link performance is underestimated, potential gain exists. Both of the above cases will lead to loss of system performance.

Currently, there are several algorithms to implement link evaluation, like Effective Exponential Signal-tonoise-ratio Mapping (EESM) [4], Mean Instantaneous Capacity (MIC) [5], Received Block Information Rate (RBIR) [6] and Mean Mutual Information per Bit (MMIB) [7]. Here RBIR and MMIB are Mean mutual Information (MI) based algorithms, with different RBIR calculation. Unfortunately, all of them are just simulation methods, lacking of theoretical analysis. Moreover, when it comes to nonlinear detection, there are still problems with all these algorithms mentioned above.

This paper proposes a universal principle for link evaluation, and extends RBIR to common wireless scenarios. Firstly, background knowledge is introduced, including models of common transmission and link evaluation; a universal principle for link evaluation is proposed; and then RBIR is extended to ERBIR with the help of this principle. Simulation results show that the proposed algorithm provides more accuracy for different scenarios. Finally, conclusions are drawn.

\section{Background}

To analyze link evaluation, common models of wireless transmission and link evaluation are presented in this section.

\subsection{Common Model of Wireless Transmission}

Following assumptions are made for analysis in this paper.

1) Multi-Input Multi-Output Orthogonal Frequency Division Multiplexing (MIMO OFDM) is adopted in wireless transmission. NT and NR indicate the number of 
transmitting and receiving antennas respectively. NOFDM indicates the number of subcarriers in OFDM symbol. As to SISO or single subcarrier case, there is $\mathrm{NT}=\mathrm{NR}=1$ or $\mathrm{NOFDM}=1$;

2) Perfect channel estimation and the channel response is flat fading on each OFDM subcarrier;

3) Detection with interference cancellation is not taken into consideration;

4) Source bits are random and iterative coding and decoding is used, for example Turbo;

5) Link evaluation interests in statistical BLock Error Rate (BLER) [1] for given CSI. Let $\mathrm{Nu}$ indicate the number of subcarriers mapped to the interested wireless resource block.

6) Modulation and Coding Scheme (MCS) levels are set to QPSK 1/2, QPSK 3/4, 16QAM 1/2, 16QAM 3/4, 64QAM 1/2, 64QAM 2/3, 64QAM 3/4 and 64QAM 5/6, referred to MCS 1 8 respectively.

Disregarding subcarrier index, MIMO OFDM transmission can be written as [2]

$$
\boldsymbol{y}=\boldsymbol{H}_{\mathrm{c}} \boldsymbol{F} \boldsymbol{x}+\boldsymbol{H}_{\mathrm{I}} \boldsymbol{x}_{\mathrm{I}}+\boldsymbol{n}
$$

where $\boldsymbol{y}$ is $\mathrm{N}_{\mathrm{R}} \times 1$ dimensional receiving signal vector; $\boldsymbol{H}_{\mathrm{c}}$ is $\mathrm{N}_{\mathrm{R}} \times \mathrm{N}_{\mathrm{T}}$ channel response matrix; $\boldsymbol{F}$ is $\mathrm{N}_{\mathrm{T}} \times \mathrm{N}_{\mathrm{S}}$ transmitting precoding matrix; $\boldsymbol{x}$ is $\mathrm{N}_{\mathrm{S}} \times 1$ independent transmitting signal vector, with unit transmitting power; $\boldsymbol{H}_{\mathrm{I}}$ is $\mathrm{N}_{\mathrm{R}} \times \mathrm{N}_{\mathrm{S}}$ interference channel response matrix; $\boldsymbol{x}_{\mathrm{I}}$ is $\mathrm{N}_{\mathrm{S}} \times 1$ independent interference signal vector, with unit transmitting power; $\boldsymbol{n}$ is $\mathrm{N}_{\mathrm{R}} \times 1$ AWGN vector, which is consisted of $\mathrm{N}_{R}$ independent AWGN elements with power of $\sigma 2$ (given $S N R, \sigma 2=10^{-S N R / 10}$ ). So this MIMO OFDM transmission is effective to

$$
\begin{gathered}
\boldsymbol{y}=\boldsymbol{H}_{\mathrm{e}} \boldsymbol{x}+\boldsymbol{n}_{\mathrm{e}} ;\left\|\boldsymbol{H}_{\mathrm{e}}\right\|_{\mathrm{F}}{ }^{2}=1 ; \mathrm{E}\left\{\boldsymbol{x} \boldsymbol{x}^{H}\right\}=\boldsymbol{I}\left(\mathrm{N}_{S}\right) ; \\
\boldsymbol{H}_{\mathrm{e}}=\boldsymbol{T}^{-1} \boldsymbol{H}_{\mathrm{c}} \boldsymbol{F} /\left\|\boldsymbol{T}^{-1} \boldsymbol{H}_{\mathrm{c}} \boldsymbol{F}\right\|_{\mathrm{F}} ; \boldsymbol{T} \boldsymbol{T}^{\mathrm{H}}=\boldsymbol{H}_{\mathrm{I}} \boldsymbol{H}_{\mathrm{I}}^{\mathrm{H}}+\sigma^{2} \boldsymbol{I}\left(\mathrm{N}_{\mathrm{R}}\right) ; \\
\mathrm{E}\left\{\boldsymbol{n}_{\mathrm{e}} \boldsymbol{n}_{\mathrm{e}}{ }^{\mathrm{H}}\right\}=\sigma_{\mathrm{e}}{ }^{2} \boldsymbol{I}\left(\mathrm{N}_{\mathrm{R}}\right) ; \sigma_{\mathrm{e}}{ }^{2}=1 /\left\|\boldsymbol{T}^{-1} \boldsymbol{H}_{\mathrm{c}} \boldsymbol{F}\right\|_{\mathrm{F}}{ }^{2}
\end{gathered}
$$

See Appendix A for a proof. Here $\boldsymbol{H}$ e is $\mathrm{N}_{\mathrm{R}} \times \mathrm{N}_{\mathrm{S}}$ effective channel response matrix. $\|\boldsymbol{A}\|_{\mathrm{F}}$ refers to the Frobenius norm of matrix $\boldsymbol{A} . \boldsymbol{I}(\mathrm{N})$ is $\mathrm{N} \times \mathrm{N}$ identity matrix. $\sigma_{\mathrm{e}}{ }^{2}$ is effective AWGN power.

\subsection{Detection Algorithms}

Consider detection at receiver. There are mainly three types of detection algorithms [8]: Minimum Mean Square Error (MMSE), Zero Forcing (ZF) and Maximum Likelihood (ML). Since MMSE and ZF are homologous, MMSE and ML are emphasized, and $\mathrm{ZF}$ is similar to MMSE.

For MMSE detection, the output signal is

$$
\boldsymbol{x}_{\mathrm{o}}=\boldsymbol{M y}=\boldsymbol{M}\left(\boldsymbol{H}_{\mathrm{e}} \boldsymbol{x}+\boldsymbol{n}_{\mathrm{e}}\right)
$$

where $\boldsymbol{M}$ is $\mathrm{N}_{\mathrm{S}} \times \mathrm{N}_{\mathrm{R}}$ dimensional equalizing matrix. Then this MIMO transmission can be divided into NS SISO transmissions with NS different Output Signal to Inter- ference and Noise Ratio (OSINR), written as $\gamma_{i}, i=1$, $2, \ldots, \mathrm{NS}$.

$$
x_{\mathrm{o}}(i)=x(i)+n(i)
$$

where $n(i)$ is independent AWGN with power of $1 / \gamma_{i}$. For MMSE, $\boldsymbol{M}$ and OSINR for each output signal are detailed in Appendix B.

As to ML detection, let $\Omega(\boldsymbol{x})$ mean the vector aggregate of every possible value of $\boldsymbol{x}$, then output signal is

$$
\mathbf{x}_{\mathrm{o}}=\underset{\mathrm{x} \in \Omega(\mathrm{x})}{\arg } \max \frac{P(\mathbf{y} \mid \mathbf{x})}{\sum_{\mathrm{q} \in \Omega(\mathrm{x})} P(\mathbf{q}) P(\mathrm{y} \mid \mathbf{q})}
$$

Note that there is an exception of Alamouti MIMO. Only one symbol can be transmitted by each transmission for Alamouti MIMO. This Alamouti MIMO is effective to SISO transmission [8], where $n$ is AWGN with power of $\sigma^{2}$.

$$
y=\|\boldsymbol{H}\| \mathrm{F}^{2} x+n
$$

For both linear and nonlinear detection, the iterative coding and decoding is adopted. The implementation of such system is described in reference [9].

\subsection{Common Model of Link Evaluation}

There have been already several algorithms to carry out link evaluation, such like EESM, MIC, RBIR and MMIB. Common model of link evaluation is shown as the following figure.

Link evaluation follows these procedures:

Step 1: Channel estimation outputs CSI of this block;

Step 2: According link evaluation algorithm, indicator $S_{k}$ for the $k^{\text {th }}$ subcarrier is computed from CSI;

Step 3: Compute average $S$ with all these indicators;

Step 4: Once the relation between $S$ and $B L E R$ of this block is definite, $B L E R$ is computed from $S$, without Monte Carlo simulation.

If necessary, Packet Error Rate (PER), Frame Error Rate (FER) and so on can be computed also, using bellowing equation [1]

$$
P E R \text { or } F E R=1-\prod_{m=1}^{N_{\mathrm{B}}}\left(1-B L E R_{m}\right)
$$

Here $\mathrm{N}_{\mathrm{B}}$ is the number of blocks in the packet or frame.

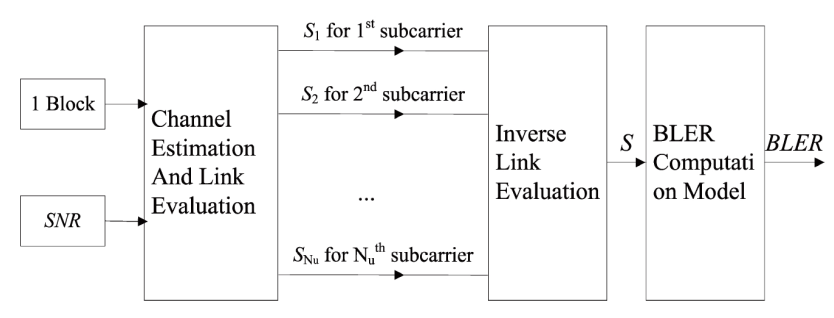

Figure 1. Common model of link evaluation 


\section{Universal Principle of Link Evaluation}

EESM, MIC, RBIR and MMIB algorithms are developed by simulation method, without strict theoretical deduction. A universal principle of link evaluation is proposed in this section, making them clear.

\subsection{Mathematical Model of Link Evaluation}

Since link evaluation mainly interests in BLER for given CSI, it should be deduced from block transmission error rate. As the transmitting block is consisted of $\mathrm{N}_{\mathrm{u}}$ subcarriers, the uncoded BLER is computed as

$$
B L E R_{u}=1-\prod_{k=1}^{\mathrm{N}_{u}}\left(1-S E R_{k}\right)=1-\left(1-S E R_{\text {ave }}\right)^{\mathrm{N}_{u}}
$$

Here $B L E R_{\mathrm{u}}$ means the statistical uncoded BLER. With the help of information theory, there is lemma 1 : When MCS of the transmitted block is given, BLER is one-one to the $B L E R_{\mathrm{u}}$. Written as

$$
B L E R=\text { MappingFunction }_{\mathrm{MCS}}\left(B L E R_{\mathrm{u}}\right)
$$

See Appendix $C$ for a proof. Then the universal principle of link evaluation is described as: find a unified and accurate indicator to reflect the BLER of the current transmitting block.

\subsection{Current Link Evaluations}

Generally speaking, there are three indicators which reflect the Symbol Error Rate (SER) under given CSI. They are OSINR, Channel Capacity and MI. Current link evaluation falls into EESM, MIC and RBIR algorithms.

\subsubsection{EESM Link Evaluation}

As $\gamma_{k}$ is known, the Chernoff limit of $S E R_{k}$ is approximated as [8]

$$
S E R_{k} \approx \exp (-\gamma k / \beta)
$$

where $\beta$ is MCS related parameter. Use the mathematical average of all $S E R_{k}$ to approximate $S E R_{\text {ave }}$ of this block, then

$$
S E R_{\mathrm{ave}} \approx \frac{1}{\mathrm{~N}_{\mathrm{u}}} \sum_{k=1}^{\mathrm{N}_{\mathrm{u}}} S E R_{k} \approx \exp \left(-\gamma_{\mathrm{eff}} / \beta\right)
$$

Then there is

$$
B L E R_{\mathrm{u}}=1-\left[1-\exp \left(-\gamma_{\mathrm{eff}} / \beta\right)\right]^{\mathrm{N}_{\mathrm{u}}}
$$

And

$$
\gamma_{\text {eff }}=-\beta \log _{\mathrm{e}}\left(\frac{1}{\mathrm{~N}_{\mathrm{u}}} \sum_{k=1}^{\mathrm{N}_{\mathrm{u}}} \exp \left(-\gamma_{k} / \beta\right)\right)
$$

The effective OSINR defined by (13) is exactly the same as in EESM [4]. The mapping function between $\gamma_{\text {eff }}$ and $B L E R$, and parameter $\beta$ can be decided by training data from Link Level Simulation (LLS).

\subsubsection{MIC Link Evaluation}

Since channel response $\boldsymbol{H}_{k}$ and $S N R$ is given, the channel capacity for this transmission is

$$
C_{k}=\frac{1}{\mathrm{~N}_{\mathrm{R}}} \log _{2}\left|\boldsymbol{I}+\frac{1}{\sigma^{2}} \boldsymbol{H}_{k} \boldsymbol{H}_{k}{ }^{H}\right|
$$

Here $|\boldsymbol{A}|$ means the determinant of matrix $\boldsymbol{A}$. The capacity decides the lower bound of $S E R_{k}$ [5], so

$$
S E R_{\mathrm{k}} \leq 1-2^{A C_{k}+B}
$$

where $A$ and $B$ are MCS related parameters. Then

$$
\begin{aligned}
& B L E R_{\mathrm{u}} \approx 1-\prod_{k=1}^{\mathrm{N}_{\mathrm{u}}}\left(1-S E R_{k}\right)=1-\prod_{k=1}^{\mathrm{N}_{\mathrm{u}}}\left(1-1+2^{A C_{k}+B}\right) \\
& =1-2^{A \sum_{k=1}^{\mathrm{N}_{\mathrm{u}}} C_{k}+\mathrm{N}_{\mathrm{u}} B}=1-2^{A \mathrm{~N}_{\mathrm{u}} M I C+\mathrm{N}_{\mathrm{u}} B}=1-2^{A_{1} M I C+A_{2}}
\end{aligned}
$$

Here $A_{1}$ and $A_{2}$ are optimized by training data from LLS, and $A 1$ and $A 2$ are listed in Table 2. Then,

$$
M I C=\frac{1}{\mathrm{~N}_{\mathrm{u}}} \sum_{k=1}^{\mathrm{N}_{\mathrm{u}}} C_{k}
$$

This is exactly the same as [5]. Then MIC is validated by the same LLS data base in previous section.

\subsubsection{RBIR Link Evaluation}

Let the transmitting symbol is $x$, and the receiving symbol is $y$ after distortion by fading channel and pollution by interference and noise. Then MI for this symbol is [10]

$$
M I=\underset{x, y}{\mathrm{E}}\left\{\log _{2} \frac{P(x, y)}{P(x) P(y)}\right\}=\underset{x, y}{\mathrm{E}}\left\{\log _{2}[1-\operatorname{SER}(x, y)]\right\}
$$

Then consider RBIR of the uncoded block

Table 1. Parameter for EESM

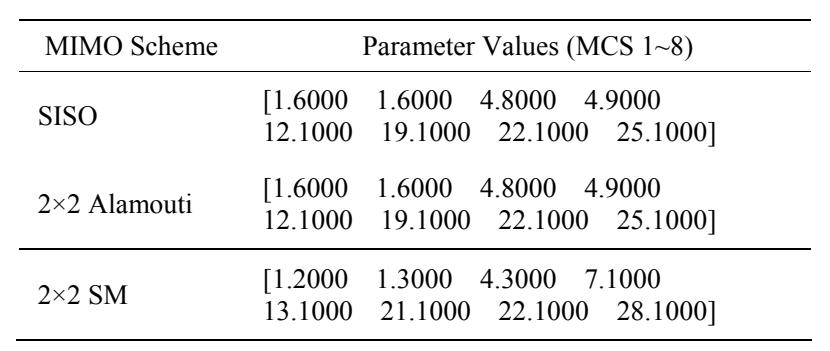

Table 2. Parameter for MIC

\begin{tabular}{ccccc}
\hline Prameter & \multicolumn{5}{c}{ Parameter Values } \\
\hline \multirow{2}{*}{$A_{1}$} & {$\left[\begin{array}{lllll}-14.3852 & -9.1091 & -8.0877 & -6.6149 \\
& -5.2316 & -4.3936 & -5.3627 & -3.3814\end{array}\right]$} \\
$A_{2}$ & {$\left[\begin{array}{lllll}18.2503 & 17.8563 & 20.6476 & 24.411 & 20.5704 \\
& 22.0257 & 28.8529 & 19.8698\end{array}\right]$} \\
\hline
\end{tabular}


$R B I R_{\mathrm{u}}=\frac{1}{\mathrm{~N}_{\mathrm{u}}} \sum_{k=1}^{\mathrm{N}_{\mathrm{u}}} M I_{k}=\frac{1}{\mathrm{~N}_{\mathrm{u}}} \sum_{k=1}^{\mathrm{N}_{\mathrm{u}}} \underset{x_{k}, y_{k}}{\mathrm{E}}\left\{\log _{2}\left[1-\operatorname{SER}\left(x_{k}, y_{k}\right)\right]\right\}$

Generally speaking, for multi-subcarriers transmission, each symbol is transmitted independently. So

$$
\begin{aligned}
R B I R_{\mathrm{u}} & =\underset{\left\{x_{k}, y_{k}\right\}}{\mathrm{E}}\left\{\frac{1}{\mathrm{~N}_{\mathrm{u}}} \sum_{k=1}^{\mathrm{N}_{\mathrm{u}}} \log _{2}\left[1-\operatorname{SER}\left(x_{k}, y_{k}\right)\right]\right\} \\
& =\underset{\left\{x_{k}, y_{k}\right\}}{\mathrm{E}}\left\{\frac{1}{\mathrm{~N}_{\mathrm{u}}} \log _{2} \prod_{k=1}^{\mathrm{N}_{\mathrm{u}}}\left[1-\operatorname{SER}\left(x_{k}, y_{k}\right)\right]\right\} \\
& =\underset{\left\{x_{k}, y_{k}\right\}}{\mathrm{E}}\left\{\frac{1}{\mathrm{~N}_{\mathrm{u}}} \log _{2} B L E R_{\mathrm{u}}\left(\left\{x_{k}, y_{k}\right\}\right)\right\}
\end{aligned}
$$

Reconsider the uncoded $B L E R_{\mathrm{u}}$

$$
R B I R_{\mathrm{u}}=\underset{\left\{x_{k}, y_{k}\right\}}{\mathrm{E}}\left\{\operatorname{RBIR}_{\mathrm{u}}\left(\left\{x_{k}, y_{k}\right\}\right)\right\}
$$

Compare (20) and (21), $R B I R_{\mathrm{u}}$ is one-one to $R B I R_{\mathrm{u}}$ of the block. From lemma $1, B L E R$ is one-one to $R B I R \mathrm{u}$ also.

According to different calculations of $R B I R_{\mathrm{u}}$, there are RBIR and MMIB algorithms.

As to RBIR, $R B I R_{\mathrm{u}}$ is computed by OSINR [6], so there are same problems as EESM, not to support ML scenario. But as it is strictly in accordance to the BLER model, RBIR shows better accuracy than EESM.

As to MMIB, computation of $R B I R_{\mathrm{u}}$ is from bit Logwise Likelihood Ratio (LLR), which is presented in reference [7], so it can support ML scenario. Also as it is strictly in accordance to the BLER model, MMIB should be of the same accuracy as RBIR.

\subsection{Principle of Link Evaluation}

There are two parts for the principle of link evaluation, based on previous analysis. Firstly, BLER should be computed from the BLER model presented before; secondly, RBIR is the most accurate indicator of BLER computation.

\section{Erbir Link Evaluation}

Previous analysis shows that RBIR reflects the transmission error probability accurately. Thus link evaluation should be based on mean mutual information indicator. This section proposes extension for RBIR, obtaining a unified and accurate ERBIR algorithm for common wireless transmissions.

\subsection{General Procedures of ERBIR Link Evaluation}

ERBIR link evaluation is implemented following these steps:

1) Get instantaneous CSI from channel estimation. The interested CSI indicators are channel response matrixes of $\left[\boldsymbol{H}_{1}, \boldsymbol{H}_{2}, \ldots, \boldsymbol{H}_{\mathrm{Nu}}\right]$, and AWGN power of $S N R$;
2) According to detection algorithms, normalized MI ' $I_{k}$ ' for each transmitted symbol is computed;

3) Average all the $I_{k}$ in this block to get RBIR;

4) Finally BLER is computed from RBIR according to RBIR to BLER mapping function which is obtained by LLS.

In Step (2), computation is the same as conventional RBIR when it comes to MMSE detection. While ML detection is used, it is not the same. So ERBIR is extension for RBIR, which is homologous to RBIR and MMIB, but providing more accurate and universal RBIR computation.

\subsection{Normalized MI Computation for SISO}

For SISO transmission, the received symbol is

$$
y=H x+n ; \mathrm{E}\left\{x x^{*}\right\}=1 ; \mathrm{E}\left\{n n^{*}\right\}=10^{-S N R / 10}
$$

The normalized MI ' $I$ ' is computed as

$$
\begin{aligned}
I & =\frac{1}{\log _{2} \mathrm{~N}_{\mathrm{QAM}}} \underset{x, y}{\mathrm{E}}\left\{\log _{2} \frac{P(x, y)}{P(x) P(y)}\right\} \\
& =\frac{1}{\log _{2} \mathrm{~N}_{\mathrm{QAM}}} \text { SISO_MI }\left(10^{-S N R / 10} /|H|^{2}\right)
\end{aligned}
$$

See Appendix D for details. And the following figure shows that it is accurate for a random selected channel ' $H$ '.

\subsection{Normalized MI Computation for MIMO}

To simplify analysis, take $2 \times 2$ MIMO as example, and analysis is similar for MIMO with more antennas. The received symbol is

$$
\left[\begin{array}{l}
y_{1} \\
y_{2}
\end{array}\right]=\left[\begin{array}{ll}
h_{11} & h_{12} \\
h_{21} & h_{22}
\end{array}\right]\left[\begin{array}{l}
x_{1} \\
x_{2}
\end{array}\right]+\left[\begin{array}{l}
n_{1} \\
n_{2}
\end{array}\right]
$$

Here assume

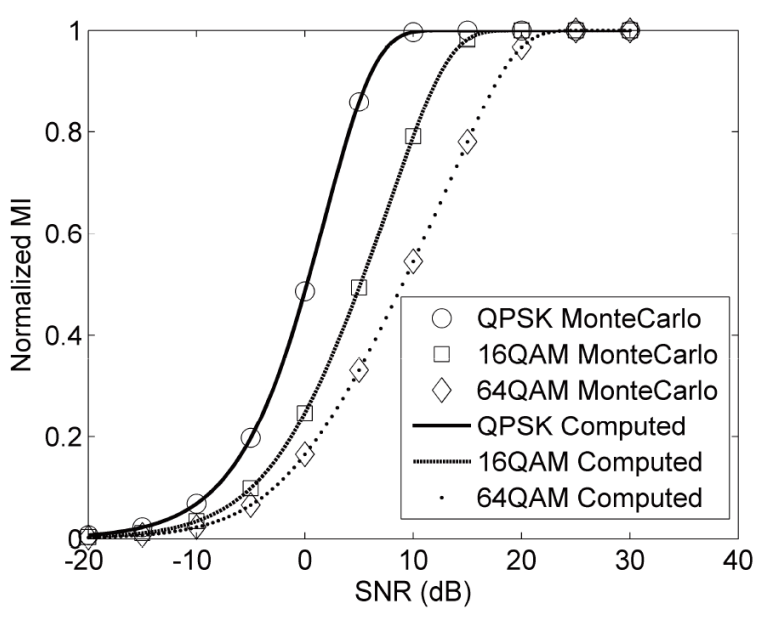

Figure 2. SISO normalized MI computation 


$$
\begin{gathered}
\left\|\left[\begin{array}{ll}
h_{11} & h_{12} \\
h_{21} & h_{22}
\end{array}\right]\right\|_{\mathrm{F}}=1 \quad\left\{\begin{array}{r}
\mathrm{E}\left\{x_{1} x_{1}^{*}\right\}=\mathrm{E}\left\{x_{2} x_{2}^{*}\right\}=1 \\
\mathrm{E}\left\{n_{1} n_{1}^{*}\right\} \mathrm{E}\left\{n_{2} n_{2}^{*}\right\}=10^{-S N R / 10}
\end{array}\right. \\
\mathrm{E}\left\{x_{1} x_{2}^{*}\right\}=0 \quad \mathrm{E}\left\{n_{1} n_{2}^{*}\right\}=0
\end{gathered}
$$

The normalized MI ' $I_{1}$ ' for the $1^{\text {st }}$ transmitted symbol is computed as

$$
\begin{gathered}
I_{1}=\frac{1}{\log _{2} \mathrm{~N}_{\mathrm{QAM}}} \underset{x, y}{\mathrm{E}}\left\{\log _{2} \frac{P\left(x_{1}, \boldsymbol{y}\right)}{P(\boldsymbol{x}) P(\boldsymbol{y})}\right\} \\
=\frac{\operatorname{VectorMI}(\boldsymbol{H}, S N R)-\mathrm{SISO}_{-} \mathrm{MI}\left(\frac{10^{-S N R / 10}}{\left|h_{12}\right|^{2}+\left|h_{22}\right|^{2}}\right)}{\log _{2} \mathrm{~N}_{\mathrm{QAM}}}
\end{gathered}
$$

Similarly

$$
I_{2}=\frac{\operatorname{VectorMI}(\boldsymbol{H}, S N R)-S \mathrm{ISO} \_\mathrm{MI}\left(\frac{10^{-S N R / 10}}{\left|h_{11}\right|^{2}+\left|h_{21}\right|^{2}}\right)}{\log _{2} \mathrm{~N}_{\mathrm{QAM}}}
$$

This computation is detailed in Appendix E. And Figure 3 shows that it is accurate for a random selected channel ' $\boldsymbol{H}$ '.

\section{Validation by Static LLS}

All algorithms for link evaluation are validated by static LLS, based on WiMAX II down link.

\subsection{Simulation Configurations}

Static LLS means that the CSI is given, and then the block transmission is trialed by a lot of Monte Carlo simulations to get real BLER under the given CSI. Then the CSI and real BLER are stored. The CSI is processed by link evaluation to get computed BLER. Obviously the

\begin{tabular}{|c|c|}
\hline Parameters & Configuration \\
\hline \multirow{3}{*}{ MIMO Scheme } & SISO/MIMO $2 \times 2$ Spatial Multiplexing $(\mathrm{SM})$ \\
\hline & Vertical EnCoding (VEC)/MIMO $2 \times 2 \mathrm{SM}$ \\
\hline & Horizontal EnCoding (HEC) \\
\hline Frame Duration & $5 \mathrm{~ms}$ \\
\hline Bandwidth & $10 \mathrm{MHz} ; \mathrm{NOFDM}=1024$ \\
\hline Channel Estimation & Ideal \\
\hline Channel Model & $\begin{array}{l}70 \% \text { ITU PedB } 3 \mathrm{kmph} \text { and } 30 \% \text { ITU VA } \\
30 \mathrm{kmph}\end{array}$ \\
\hline Channel Coding & Turbo \\
\hline MCS & MCS 5 \\
\hline Block Size & $\begin{array}{l}16 \text { subcarrier } \times 6 \text { symbol (Subcarriers are continu- } \\
\text { ously allocated in wireless resource block) }\end{array}$ \\
\hline Detection & MMSE/ML \\
\hline Link Adaptation & Disable \\
\hline
\end{tabular}
more different between real and computed BLER, the worse the link evaluation algorithm is. Configuration of static LLS is shown as the following Table 3.

Table 3. Configuration of static LLS

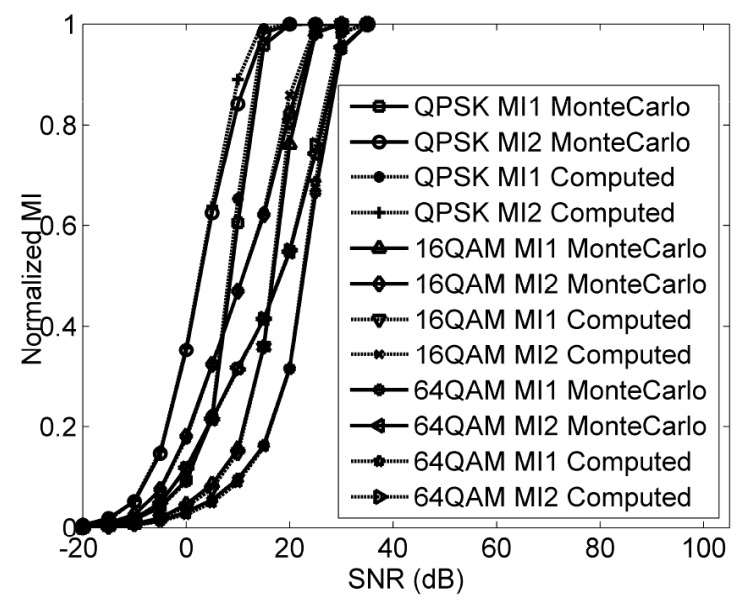

Figure 3. $2 \times 2$ Normalized MI computation

\subsection{Simulation Results}

In these results figures, the black bold curve is the computing function of indicator $S$ to BLER obtained by training data from LLS; and the marked point is plotted with real $B L E R$ and $S$ computed by the adopted link evaluation algorithm. The more deviation between the marked point and black bold curve, the more inaccurate is the link evaluation algorithm.

\subsubsection{Link Evaluation for SISO}

Firstly, SISO transmission with MMSE detection is validated by different link evaluation algorithms, shown as the following figures.

From these figures, it is obvious that although EESM, MIC and MMIB algorithms can obtain accurate enough link evaluation. RBIR/ERBIR algorithm can obtain most accurate link evaluation for simulated transmission Monte Carlo trials. Moreover, RBIR/ERBIR algorithm doesn't need any channel related tuning parameters, which makes RBIR/ERBIR more universal.

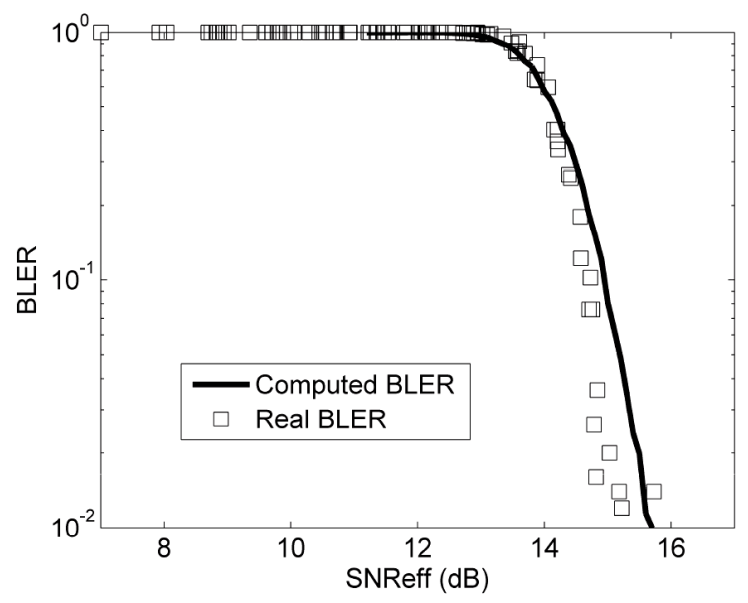

Figure 4(a). EESM LE for SISO MMSE 


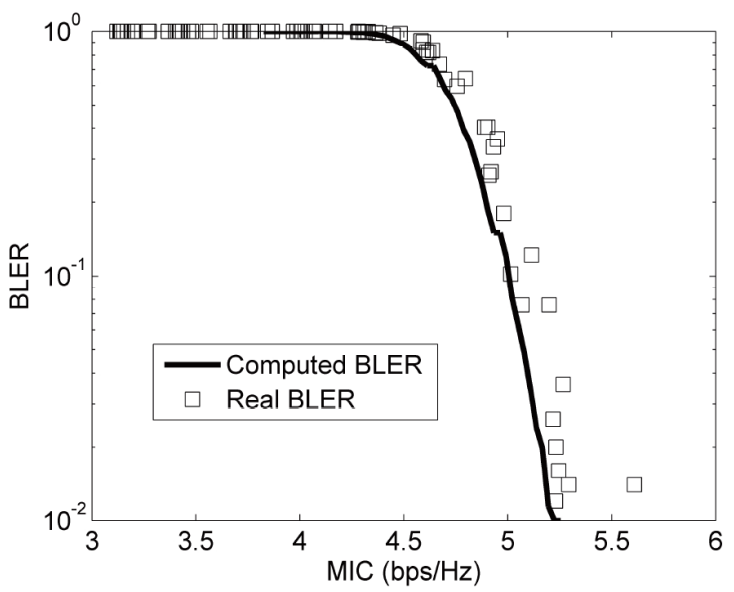

Figure 4(b). MIC LE for SISO MMSE

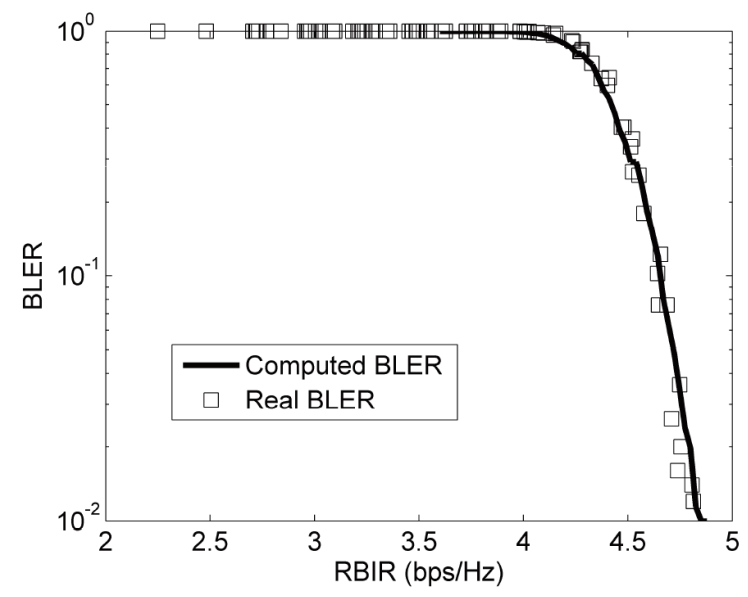

Figure 4(c). RBIR/ERBIR LE for SISO MMSE

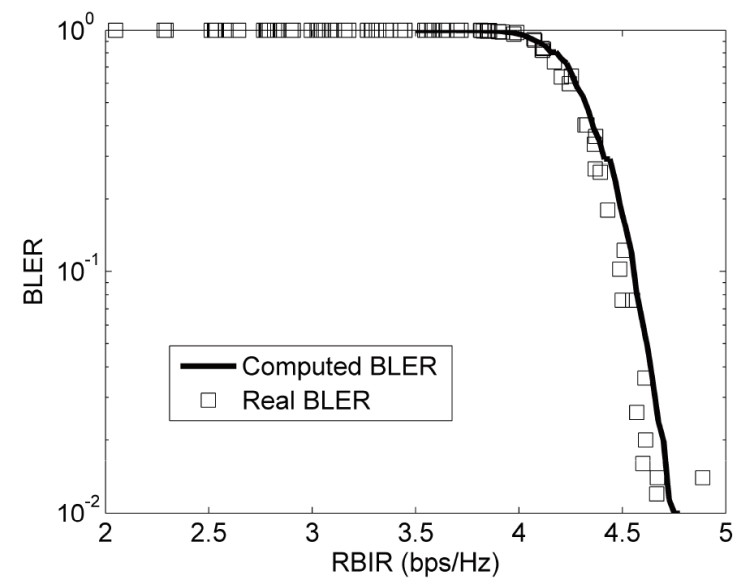

Figure 4(d). MMIB LE for SISO MMSE

Then, SISO transmission with ML detection is validated by different link evaluation algorithms, shown as the following figures.

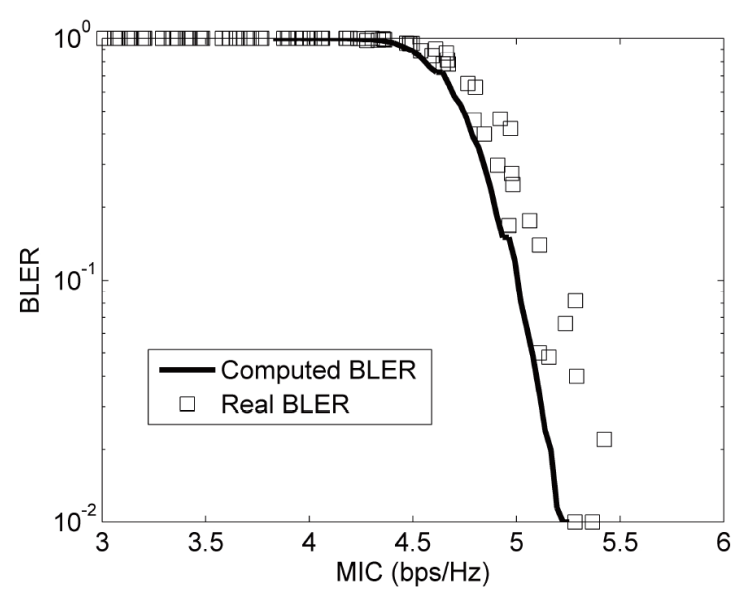

Figure 5(a). MIC LE for SISO ML

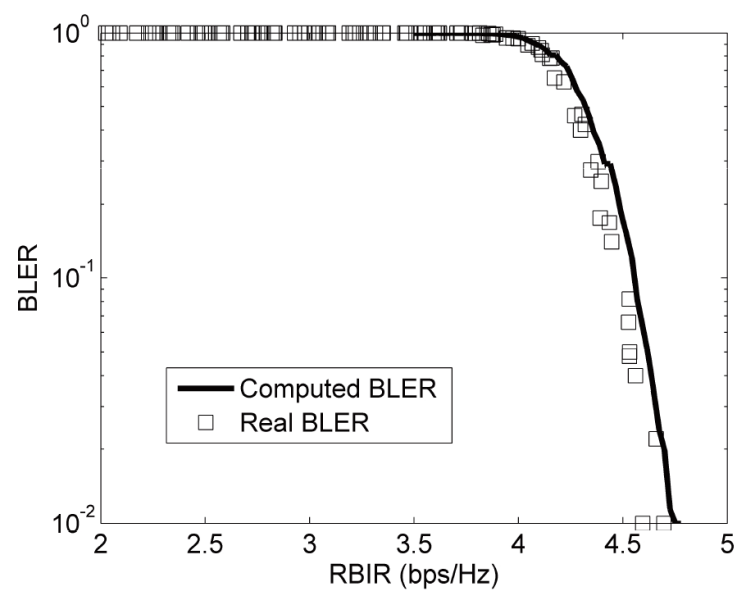

Figure 5(b). MMIB LE for SISO ML

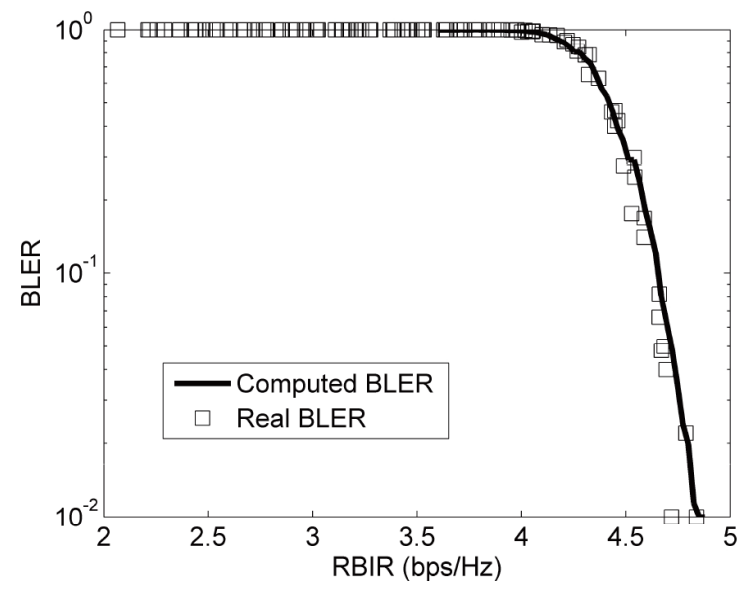

Figure 5(c). ERBIR LE for SISO ML

From these figures, it is obvious that EESM and RBIR algorithm is invalid, and MIC algorithm shows too much inaccuracy. MMIB and ERBIR are of accurate enough 


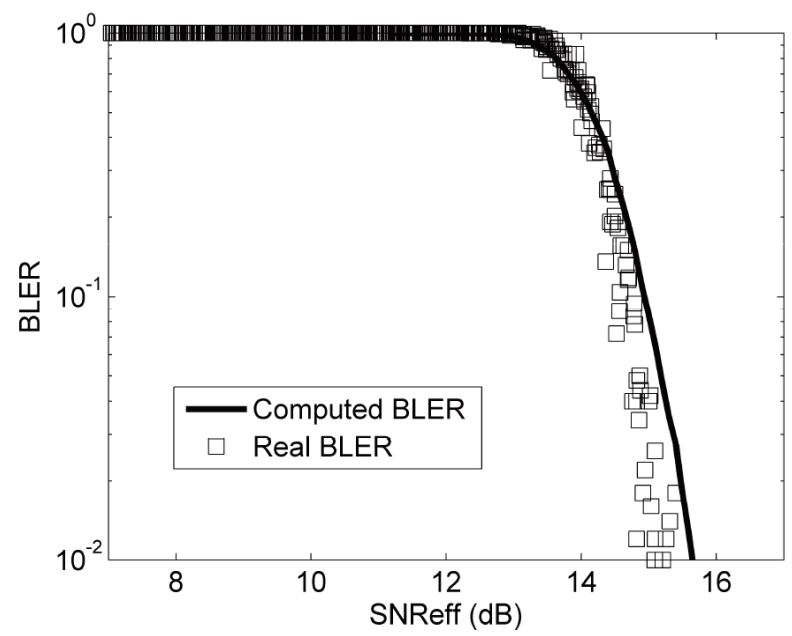

Figure 6(a). EESM LE for VEC MMSE

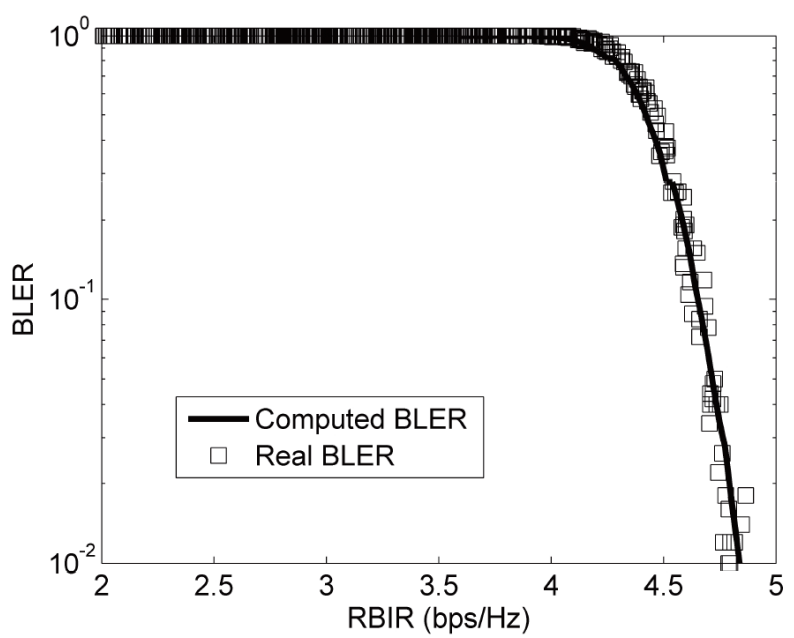

Figure 6(c). RBIR/ERBIR LE for VEC MMSE

results, while ERBIR is a bit better than MMIB.

Here simulation results also validate the theoretical conclusions. MIC chooses the upper bound of SER, so all the real BLER are bigger than computed BLER. And MMIB uses approximation in MI computation, so there is a little inaccuracy.

\subsubsection{Link Evaluation for VEC}

Firstly, VEC transmission with MMSE detection is validated by different link evaluation algorithms, shown as the following figures.

These figures show that although EESM, MIC and MMIB algorithms can also obtain quite accurate link evaluation, RBIR/ERBIR algorithm is the most accurate. Moreover, RBIR/ERBIR algorithm doesn't need any channel related tuning parameters.

Then, VEC transmission with ML detection is validated by different link evaluation algorithms, shown as

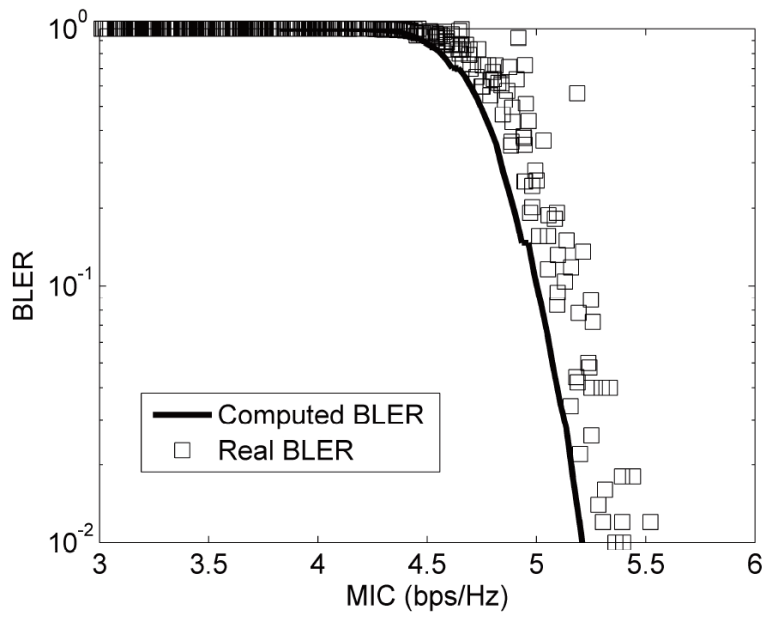

Figure 6(b). MIC LE for VEC MMSE

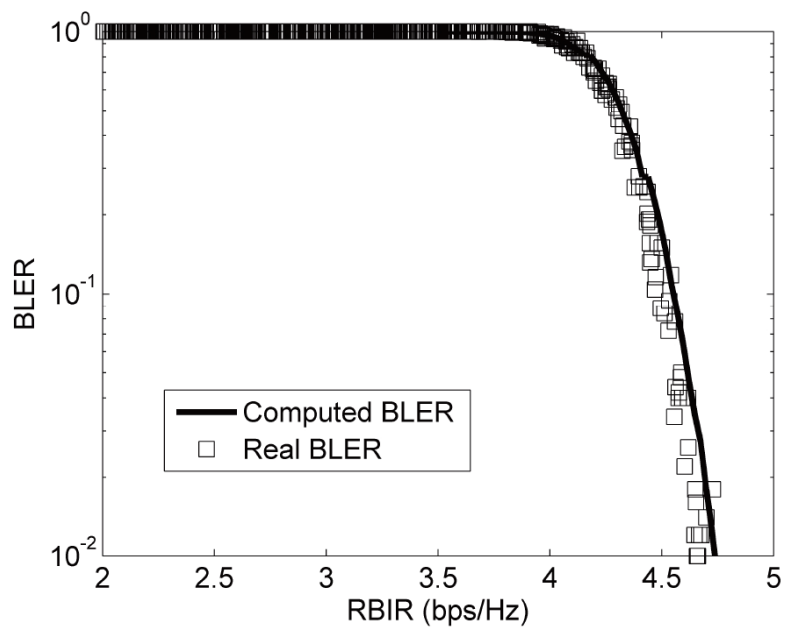

Figure 6(d). MMIB LE for VEC MMSE

the following figures.

Figure 7(a), Figure 7(b) and Figure 7(c) show that EESM and RBIR algorithms are invalid, and MIC and MMIB algorithms show too much inaccuracy. ERBIR algorithm betters the accuracy of link evaluation for VEC ML transmissions a lot, although there is still some inaccuracy.

Here, MIC algorithm only provides the upper bound of wireless transmissions, and it is of the worst accuracy. Although MMIB seems a little better, for the sake of limited parameters presented in reference [7], the RBIR is not very accurate, so MMIB shows worse results than ERBIR.

\subsubsection{Link Evaluation for HEC}

Firstly, HEC transmission with MMSE detection is validated by different link evaluation algorithms, shown as the following figures. 


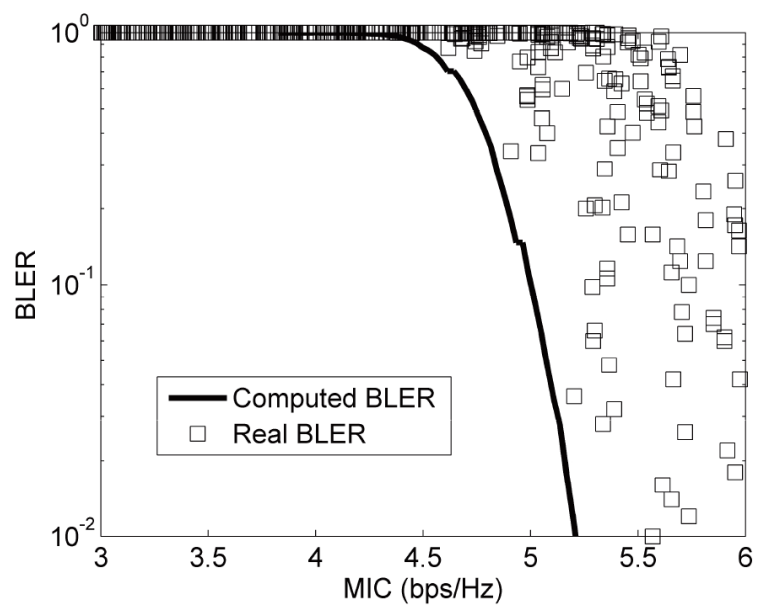

Figure 7(a). MIC LE for VEC ML

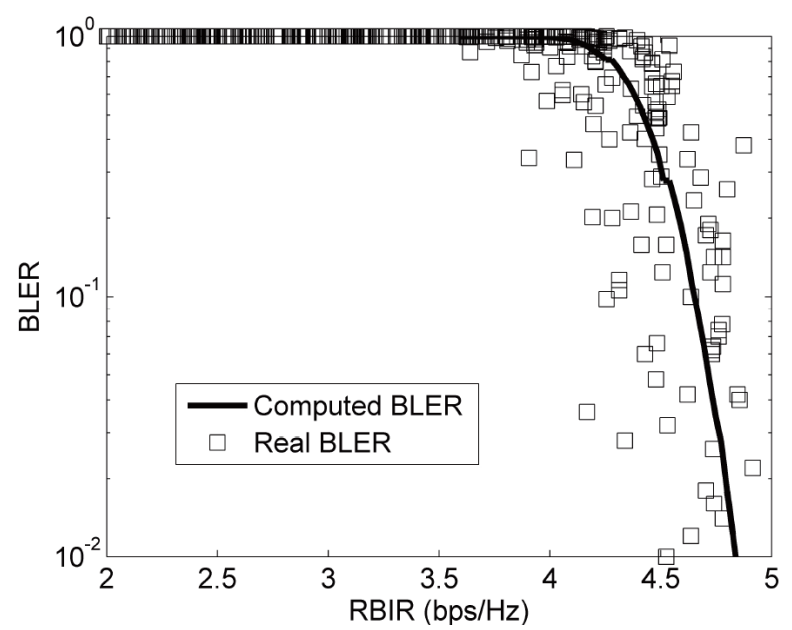

Figure 7(b). MMIB LE for VEC ML

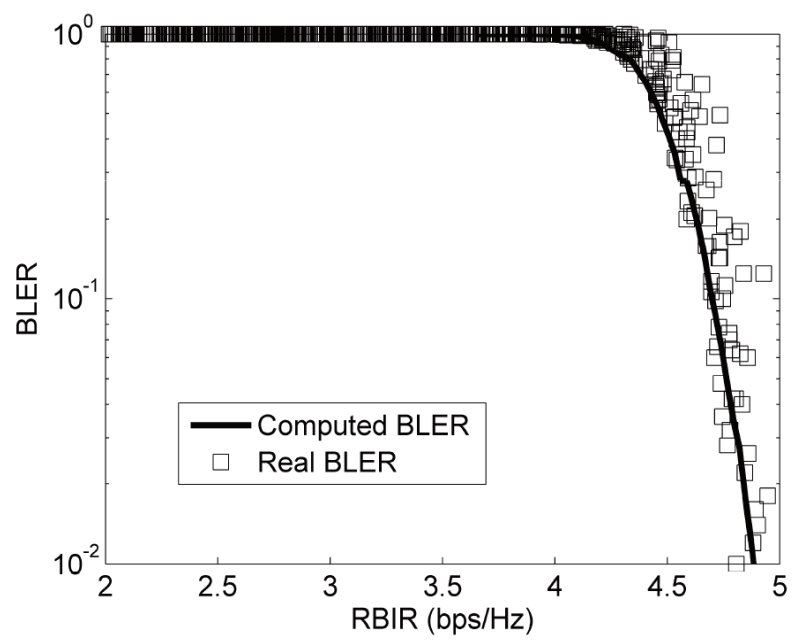

Figure 7(c). ERBIR LE for VEC ML

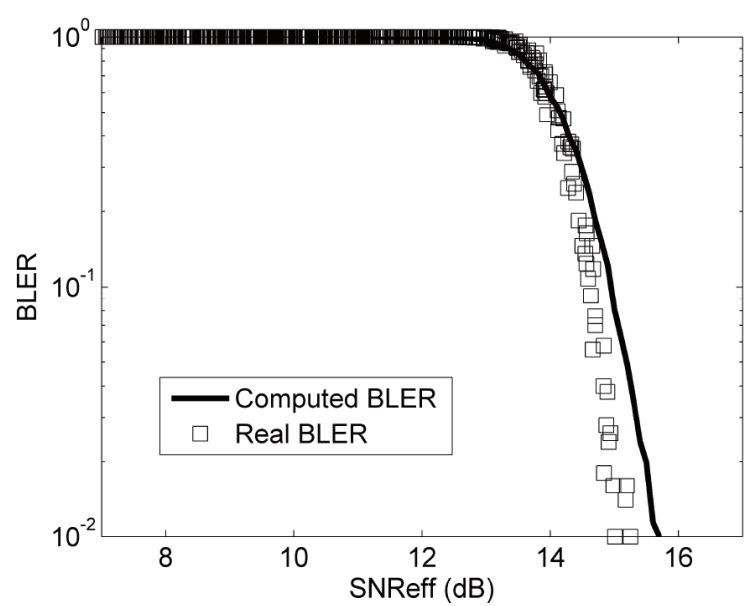

Figure 8(a). EESM LE for HEC MMSE

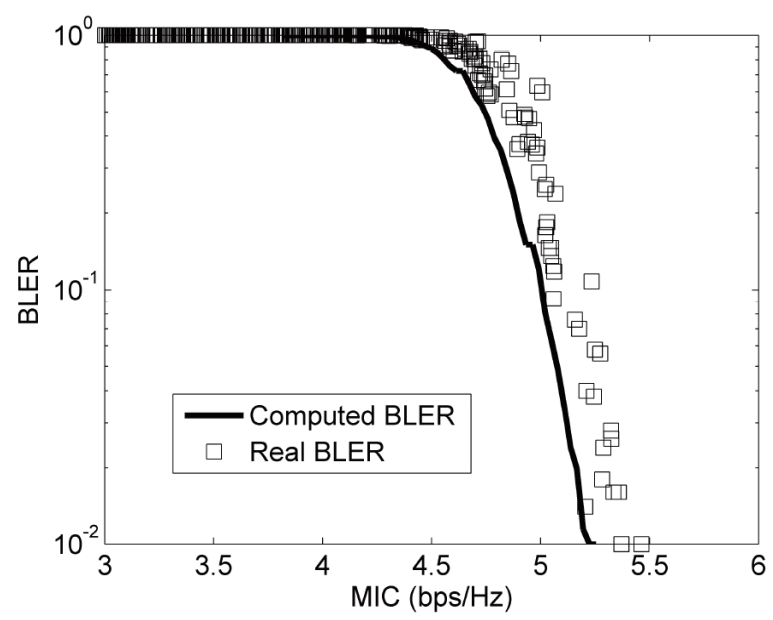

Figure 8(b). MIC LE for HEC MMSE

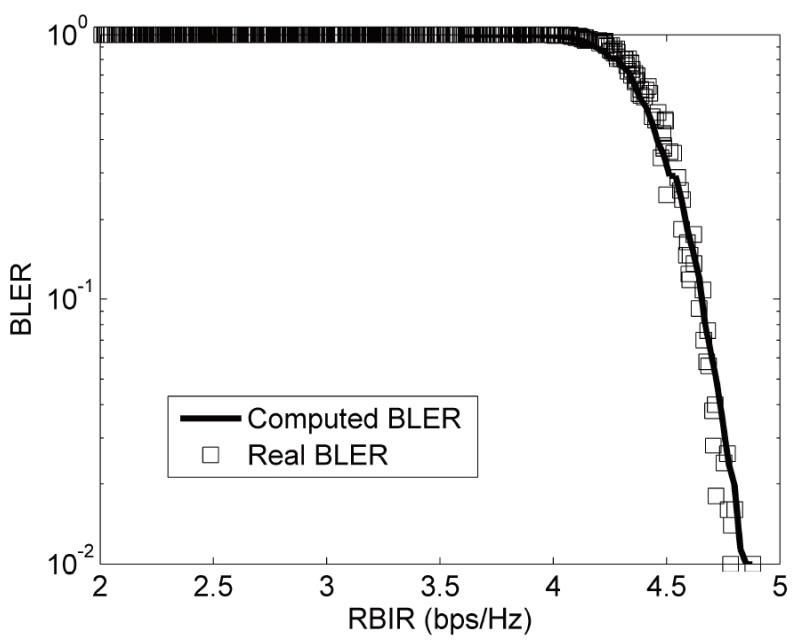

Figure 8(c). RBIR/ERBIR LE for HEC MMSE 


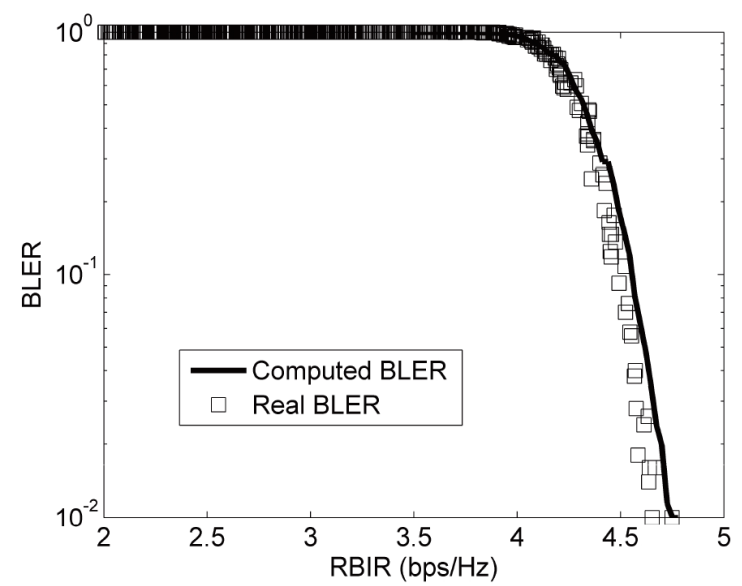

Figure 8(d). MMIB LE for HEC MMSE

These figures show that although EESM, MIC and MMIB algorithms can also obtain quite accurate link evaluation, RBIR/ERBIR algorithm is the most accurate. Moreover, RBIR/ERBIR algorithm doesn't need any channel related tuning parameters.

Then, HEC transmission with ML detection is validated by different link evaluation algorithms, shown as the following figures.

Figure 9 shows that EESM, RBIR, MIC and MMIB algorithms are invalid at all. Only ERBIR algorithm can achieve link evaluation for HEC ML transmissions.

\subsection{Further Results Comparisons and Analysis}

To ensure the universality of the simulation, more MCS levels are simulated. Following configuration in Table 3, MCS levels are set to MCS 1 8 with different MIMO schemes respectively. And the average difference is listed in the following tables. The average difference is measured by Mean Square Error Root (MSER) between computed and real BLER values.

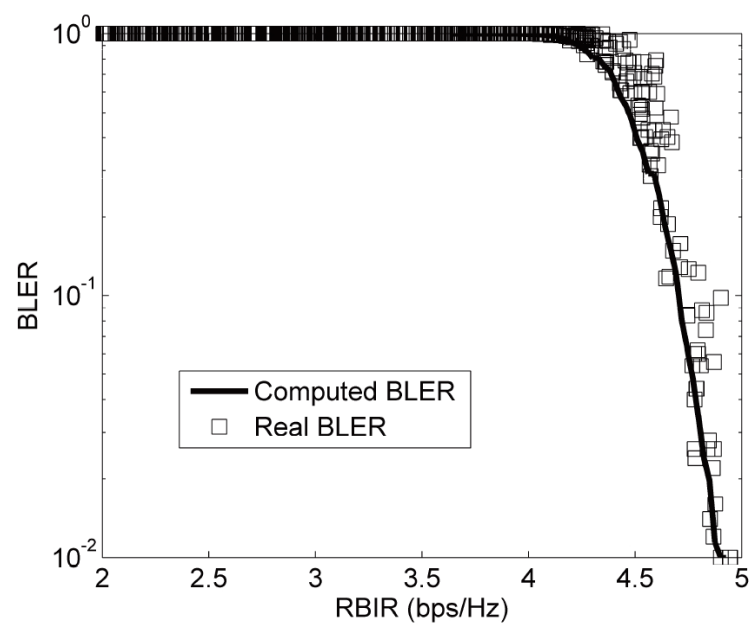

Figure 9. ERBIR LE for HEC ML
Table 4. MSER for EESM link evaluation

\begin{tabular}{|c|c|c|c|c|c|}
\hline \multirow{2}{*}{$\begin{array}{c}\text { Transmission } \\
\text { Mode }\end{array}$} & \multicolumn{5}{|c|}{ MSE } \\
\hline & EESM & MIC & RBIR & MMIB & ERBIR \\
\hline $\begin{array}{l}\text { SISO with MMSE } \\
\text { Detection }\end{array}$ & 0.0369 & 0.1295 & 0.0247 & 0.0400 & 0.0247 \\
\hline $\begin{array}{l}\text { SISO with ML } \\
\text { Detection }\end{array}$ & $\begin{array}{c}\text { Not } \\
\text { Supported }\end{array}$ & 0.1296 & $\begin{array}{c}\text { Not } \\
\text { Supported }\end{array}$ & 0.0469 & 0.0241 \\
\hline $\begin{array}{l}\text { VEC with MMSE } \\
\text { Detection }\end{array}$ & 0.0547 & 0.1348 & 0.0604 & 0.0622 & 0.0604 \\
\hline $\begin{array}{l}\text { VEC with ML } \\
\text { Detection }\end{array}$ & $\begin{array}{c}\text { Not } \\
\text { Supported }\end{array}$ & 0.3956 & $\begin{array}{c}\text { Not } \\
\text { Supported }\end{array}$ & 0.1574 & 0.0956 \\
\hline $\begin{array}{l}\text { HEC with MMSE } \\
\text { Detection }\end{array}$ & 0.0256 & 0.0851 & 0.0206 & 0.0312 & 0.0206 \\
\hline $\begin{array}{l}\text { HEC with ML } \\
\text { Detection }\end{array}$ & $\begin{array}{c}\text { Not } \\
\text { Supported }\end{array}$ & $\begin{array}{c}\text { Not } \\
\text { Supported }\end{array}$ & $\begin{array}{c}\text { Not } \\
\text { Supported }\end{array}$ & $\begin{array}{c}\text { Not } \\
\text { Supported }\end{array}$ & 0.0791 \\
\hline
\end{tabular}

As to EESM and RBIR, although they have achieved quite accurate link evaluation for wireless transmissions with MMSE detection, because the computation is based on OSINR, they can not support ML detection scenarios. To solve this problem, MIC and MMIB are developed. Unfortunately, they are not accurate for some levels either.

EESM, MIC and MMIB need MCS and CSI related tuning parameters, while RBIR does not. This makes EESM, MIC and MMIB not universal. RBIR is the most common algorithm for link evaluation, but it can be used for MMSE only. ERBIR can support link evaluation for all scenarios.

Simulation results in Table 4 show that ERBIR can provide more accurate link evaluation and more universality. Moreover, the MCS and CSI related tuning parameters are no longer necessary, which makes ERBIR become a universal and accurate method for link evaluation.

\section{Validation by Link Adaptation and SLS}

Link evaluations are validated by link adaptation and SLS of WiMAX II down link, profiling the influence caused by inaccuracy of link evaluation. Since previous results show that ERBIR is accurate, and MIC is not, link adaptation and SLS with ERBIR and MIC link evaluations are implemented.

\subsection{Validation by Link Adaptation}

Basic configuration of dynamical LLS is the same as Table 2, with link adaptation enable, $2 \times 2$ Alamouti STBC and MIMO $2 \times 2$ SM VEC of all MCS levels adaptation, and ML detection. Receiver dynamically estimates the statistical performance of wireless channel, and chooses the MCS level which can get best Spectrum Efficiency (SE) and acceptable BLER, then feeds it back to the transmitter [1].

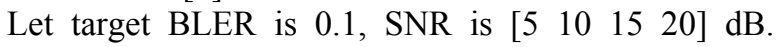
Firstly Hybrid Automatic Repeat reQuest (HARQ) is disabled, and simulation results are listed in the following Table 5. 
Table 5. Dynamical LLS results without HARQ

\begin{tabular}{lcc}
\hline & Extended MI & MIC \\
\hline BLER & {$[0.0334,0.0125$,} & {$[0.1545,0.1482$,} \\
& $0.0052,0.047]$ & $0.19,0.2495]$ \\
Throughput $\left(10^{3}\right.$ bits $)$ & {$[0.721,1.111$,} & {$[0.687,1.082$,} \\
Total Retransmission & $2.007,3.093]$ & $1.819,2.652]$ \\
Times & {$\left[\begin{array}{llll}0 & 0 & 0 & 0\end{array}\right]$} & {$\left[\begin{array}{lll}0 & 0 & 0\end{array}\right]$}
\end{tabular}

Then enable HARQ with maximum retransmission times of 3. Simulation results are listed in the following Table 6.

Compare the results of link adaptation with/without HARQ, it is obvious that accurate ERBIR link evaluation will ensure wireless system to choose proper MCS level, obtaining better BLER and throughput, and reducing the retransmission times. While using inaccurate MIC link evaluation, it is shown that MIC will overestimate the link performance, as shown in simulation results in previous section. So BLER and retransmission times increase, and throughput decreases.

\subsection{Validation by SLS}

Configuration of dynamical SLS is listed in Table 6. In SLS, link evaluation is used to hold down real coding and decoding procedures, reducing SLS complexity, as described in Reference [1]. Because the BLER in SLS is computed by link evaluation, the SLS results will become

Table 6. Dynamical LLS results with HARQ

\begin{tabular}{|c|c|c|}
\hline & Extended MI & $\mathrm{MIC}$ \\
\hline BLER & {$\left[\begin{array}{llll}0 & 0 & 0 & 0\end{array}\right]$} & {$\left[\begin{array}{llll}0 & 0 & 0 & 0\end{array}\right]$} \\
\hline Throughput $\left(10^{3}\right.$ bits $)$ & $\begin{array}{c}{[0.747,1.382,} \\
2.294,3.476]\end{array}$ & $\begin{array}{l}{[0.725,1.166,} \\
2.023,3.245]\end{array}$ \\
\hline $\begin{array}{l}\text { Total Retransmission } \\
\text { Times }\end{array}$ & {$[13,5,3,9]$} & {$[53,86,46,53]$} \\
\hline
\end{tabular}

Table 7. Configuration of SLS

\begin{tabular}{ll}
\hline \multicolumn{1}{c}{ Parameters } & \multicolumn{1}{c}{ Configuration } \\
\hline MIMO Scheme & Single user, $2 \times 2$ Alamouti STBC and \\
Frame Duration & $5 \mathrm{~ms}$ \\
Bandwidth & $10 \mathrm{MHz}$; NOFDM $=1024$ \\
Channel Estimation & ideal \\
Channel Model & $70 \%$ ITU PedB 3kmph and 30\% ITU \\
Channel Coding & VA 30kmph \\
& Turbo \\
MCS & QPSK 1/2; QPSK 3/4; 16QAM 1/2; \\
& $16 Q A M 3 / 4 ; 64 Q A M ~ 1 / 2 ; 64 Q A M ~ 2 / 3 ;$ \\
Block Size & 64QAM 3/4; 64QAM 5/6; \\
& 16 subcarrier×6 symbol (Subcarriers are \\
Detection & continuously allocated in wire- less re- \\
Link Adaptation & source block) \\
HARQ & ML \\
Target BLER & Enable \\
Link Evaluation & Enable, with maximum retransmission \\
Cell Configuration & times of 3 \\
Scheduling & 0.1 \\
\hline
\end{tabular}

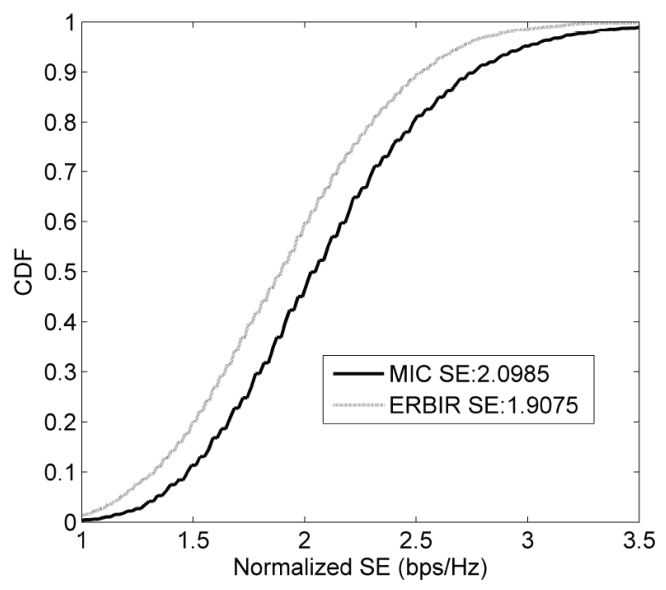

Figure 10. CDF of SLS SE

inaccurate when link evaluation can not provide accurate BLER.

Figure 10 shows the Cumulative Distribution Function (CDF) of SLS SE results. It is shown that the SLS SE is overestimated by MIC link evaluation by (2.0985 $1.9075) / 1.9075 \times 100 \% \approx 10 \%$. It is obvious that inaccurate link evaluation will lead to incredible SLS results.

\section{Conclusions}

Link evaluation aims to provide a fading insensitive performance metric for common transmissions. It is proven from the view of information theory that RBIR is the most accurate metric, and a method to compute RBIR from CSI is proposed. Simulation results of LLS and SLS show that the proposed ERBIR algorithm works very well for common transmissions, solving the problems existing in current link evaluations.

\section{Acknowledgments}

Thanks to Tan Zhenhui, Zheng Hongming, and Chen Yueyun for their great support.

This work is supported in part by the Hi-tech research and development program of China (2007AA01Z277), National Natural Science Foundation of China (6077 2035), University Doctorial Foundation of China (2007 0004010), and Intel China Research Centre.

\section{REFERENCES}

[1] IEEE 802.16 Broadband Wireless Access Working Group. Draft IEEE $802.16 \mathrm{~m}$ Evaluation Methodology Document. http:// ieee802.org/16/.July 18th, 2008.

[2] $\mathrm{HE} \mathrm{X,} \mathrm{NIU} \mathrm{K,} \mathrm{HE} \mathrm{Z} \mathrm{Q,} \mathrm{et} \mathrm{al.} \mathrm{Link} \mathrm{layer} \mathrm{abstraction} \mathrm{in}$ MIMO-OFDM system. International Workshop on Cross Layer Design, IWCLD’07, Sept. 20-21, 2007: 41-44.

[3] AKYILDIZ F, LEE W Y, AND VURAN M C, et al. A survey on spectrum management in cognitive radio networks. IEEE Communication Magazine, Apr. 2008, 46(4): 40-48.

[4] 3GPP TSG-RAN-1 Meeting \#35, Effective SIR Computation for 
OFDM System-Level Simulations.

[5] WiMAX Forum. Mobile WiMAX-Part I: A Technical Overview and Performance Evaluation. white paper, August 2006.

[6] Ericsson. A Fading-Insensitive Performance Metric for a Unified Link Quality Model.

[7] SAYANA K, ZHUANG J. Link performance abstraction based on mean mutual information per bit (MMIB) of the LLR channel. http://ieee802.org/16/. May 2nd, 2008.

[8] PAULRAJ A. Introduction to space-time wireless communications. 1st Edition. Cambridge University Press, England, 2003:

\section{APPENDIX}

\section{Effective MIMO Transmission}

The original wireless transmission is $\boldsymbol{y}=\boldsymbol{H}_{\mathrm{c}} \boldsymbol{F} \boldsymbol{x}+\boldsymbol{H}_{\mathrm{I}} \boldsymbol{x}_{\mathrm{I}}+\boldsymbol{n} ; \mathrm{E}\{\boldsymbol{x} \boldsymbol{x} \mathrm{H}\}=\boldsymbol{I}(\mathrm{NS}) ; \mathrm{E}\left\{\boldsymbol{x}_{\mathrm{I}} \boldsymbol{x}_{\mathrm{I}}^{\mathrm{H}}\right\}=\boldsymbol{I}\left(\mathrm{N}_{\mathrm{S}}\right) ;$

$$
\mathrm{E}\left\{\boldsymbol{n} \boldsymbol{n}^{\mathrm{H}}\right\}=\sigma^{2} \boldsymbol{I}\left(\mathrm{N}_{\mathrm{R}}\right)
$$

Because receiver knows nothing about interference symbol but the correlation, and $\boldsymbol{H}_{\mathrm{I}}$ is consisted of correlated Gaussians, $\boldsymbol{H}_{\mathrm{I}} \boldsymbol{x}_{\mathrm{I}}+\boldsymbol{n}$ is approximated as correlated Gaussians. Assume $\boldsymbol{R}_{\mathrm{I}}$ is known to the receiver.

$$
\mathrm{E}\left\{\left(\boldsymbol{H}_{\mathrm{I}} \boldsymbol{x}_{\mathrm{I}}+\boldsymbol{n}\right)\left(\boldsymbol{H}_{\mathrm{I}} \boldsymbol{x}_{\mathrm{I}}+\boldsymbol{n}\right)^{\mathrm{H}}\right\}=\boldsymbol{R}_{\mathrm{I}}+\sigma^{2} \boldsymbol{I}\left(\mathrm{N}_{\mathrm{R}}\right)
$$

Let $\mathrm{E}\left\{\boldsymbol{n}_{1} \boldsymbol{n}_{1}{ }^{\mathrm{H}}\right\}=\boldsymbol{I}\left(\mathrm{N}_{\mathrm{R}}\right)$, and $\boldsymbol{T} \boldsymbol{T}^{\mathrm{H}}=\boldsymbol{R}_{\mathrm{I}}+\sigma^{2} \boldsymbol{I}\left(\mathrm{N}_{\mathrm{R}}\right)$. So

$$
\mathrm{E}\left\{\left(\boldsymbol{T} \boldsymbol{n}_{1}\right)\left(\boldsymbol{T} \boldsymbol{n}_{1}\right)^{\mathrm{H}}\right\}=\mathrm{E}\left\{\left(\boldsymbol{H}_{\mathrm{I}} \boldsymbol{x}_{\mathrm{I}}+\boldsymbol{n}\right)\left(\boldsymbol{H}_{\mathrm{I}} \boldsymbol{x}_{\mathrm{I}}+\boldsymbol{n}\right)^{\mathrm{H}}\right\}
$$

This means $\boldsymbol{T} \boldsymbol{n}_{1}$ is effective to $\boldsymbol{H}_{\mathrm{I}} \boldsymbol{x}_{\mathrm{I}}+\boldsymbol{n}$, so the original wireless transmission is effective to

$$
\boldsymbol{y}=\boldsymbol{H c} \boldsymbol{F} \boldsymbol{x}+\boldsymbol{T} \boldsymbol{n}_{1} ; \mathrm{E}\left\{\boldsymbol{x} \boldsymbol{x}^{\mathrm{H}}\right\}=\boldsymbol{I}\left(\mathrm{N}_{\mathrm{S}}\right) ; \mathrm{E}\left\{\boldsymbol{n}_{1} \boldsymbol{n}_{1}{ }^{\mathrm{H}}\right\}=\boldsymbol{I}\left(\mathrm{N}_{\mathrm{R}}\right)
$$

Consider identical transform, it is effective to

$$
\boldsymbol{y}=\left(\boldsymbol{T}^{-1} \boldsymbol{H}_{\mathrm{c}} \boldsymbol{F} \boldsymbol{x}+\boldsymbol{n}_{1}\right) /\left\|\boldsymbol{T}^{-1} \boldsymbol{H}_{\mathrm{c}} \boldsymbol{F}\right\|_{\mathrm{F}}=\boldsymbol{H}_{\mathrm{e}} \boldsymbol{x}+\boldsymbol{n}_{\mathrm{e}}
$$

Where

$$
\begin{gathered}
\boldsymbol{H}_{\mathrm{e}}=\boldsymbol{T}^{-1} \boldsymbol{H}_{\mathrm{c}} \boldsymbol{F} /\left\|\boldsymbol{T}^{-1} \boldsymbol{H}_{\mathrm{c}} \boldsymbol{F}\right\|_{\mathrm{F}} ; \mathrm{E}\left\{\boldsymbol{n}_{\mathrm{e}} \boldsymbol{n}_{\mathrm{e}}{ }^{\mathrm{H}}\right\}=\sigma_{\mathrm{e}}{ }^{2} \boldsymbol{I}\left(\mathrm{N}_{\mathrm{R}}\right) ; \\
\sigma_{\mathrm{e}}{ }^{2}=1 /\left\|\boldsymbol{T}^{-1} \boldsymbol{H}_{\mathrm{c}} \boldsymbol{F}\right\|_{\mathrm{F}}
\end{gathered}
$$

This identity between (28) and (33) is proven from the view of capacity. Let $|\boldsymbol{A}|$ means the determinant of matrix $\boldsymbol{A}$. Channel capacity of the original transmission is

$$
\begin{aligned}
C_{1} & =\log _{2}\left|\pi \mathrm{eE}\left\{\boldsymbol{y y}^{\mathrm{H}}\right\}\right|-\log _{2}\left|\pi \mathrm{eE}\left\{\left(\boldsymbol{H}_{\mathrm{I}} \boldsymbol{x}_{\mathrm{I}}+\boldsymbol{n}\right)\left(\boldsymbol{H}_{\mathrm{I}} \boldsymbol{x}_{\mathrm{I}}+\boldsymbol{n}\right)^{\mathrm{H}}\right\}\right| \\
& =\log _{2}\left|\frac{\mathrm{E}\left\{\left(\boldsymbol{H}_{c} \boldsymbol{F} x+\boldsymbol{H}_{\mathrm{I}} \boldsymbol{x}_{\mathrm{I}}+\boldsymbol{n}\right)\left(\boldsymbol{H}_{c} \boldsymbol{F} \boldsymbol{x}+\boldsymbol{H}_{\mathrm{I}} \boldsymbol{x}_{\mathrm{I}}+\boldsymbol{n}\right)^{\mathrm{H}}\right\}}{\mathrm{E}\left\{\left(\boldsymbol{H}_{\mathrm{I}} \boldsymbol{x}_{\mathrm{I}}+\boldsymbol{n}\right)\left(\boldsymbol{H}_{\mathrm{I}} \boldsymbol{x}_{\mathrm{I}}+\boldsymbol{n}\right)^{\mathrm{H}}\right\}}\right|
\end{aligned}
$$

86-88, 178-198.

[9] CHUL H K, SUNGWOO P, MOON J, et al. Iterative joint detection and decoding for MIMO-OFDM wireless communications. Fortieth Asilomar Conference on Signals, Systems and Computers, ACSSC'06. Oct.-Nov. 2006, 1752-1756.

[10] SHANNON C. E. A mathematical theory of communication. The Bell System Technical Journal, July, October, 1948, 27: 379-423, 623-656.

[11] LEE J. W, BLAHUT R. E. Generalized EXIT chart and BER analysis of finite-length turbo codes. Global Telecommunication Conference, Dec. 2003, 4(1-5): 2067-2072.

$$
\begin{aligned}
& =\log _{2}\left|\frac{\boldsymbol{H}_{c} \boldsymbol{F} \boldsymbol{F}^{\mathrm{H}} \boldsymbol{H}_{c}^{\mathrm{H}}+\boldsymbol{R}_{\mathrm{I}}+\sigma^{2} \boldsymbol{I}\left(\mathrm{N}_{R}\right)}{\boldsymbol{R}_{\mathrm{I}}+\sigma^{2} \boldsymbol{I}\left(\mathrm{N}_{R}\right)}\right| \\
& =\log _{2}\left|\frac{\boldsymbol{T}^{-1}\left[\boldsymbol{H}_{c} \boldsymbol{F} \boldsymbol{F}^{\mathrm{H}} \boldsymbol{H}_{c}{ }^{\mathrm{H}}+\boldsymbol{R}_{\mathrm{I}}+\sigma^{2} \boldsymbol{I}\left(\mathrm{N}_{\mathrm{R}}\right)\right]\left(\boldsymbol{T}^{\mathrm{H}}\right)^{-1}}{\boldsymbol{T}^{-1}\left[\boldsymbol{R}_{\mathrm{I}}+\sigma^{2} \boldsymbol{I}\left(\mathrm{N}_{\mathrm{R}}\right)\right]\left(\boldsymbol{T}^{\mathrm{H}}\right)^{-1}}\right| \\
& =\log _{2}\left|\boldsymbol{I}\left(\mathrm{N}_{\mathrm{R}}\right)+\left(\boldsymbol{T}^{-1} \boldsymbol{H}_{\mathrm{c}} \boldsymbol{F}\right)\left(\boldsymbol{T}^{-1} \boldsymbol{H}_{\mathrm{c}} \boldsymbol{F}\right)^{\mathrm{H}}\right|
\end{aligned}
$$

Then the channel capacity of the effective transmission is

$$
C 2=\log _{2}\left|\boldsymbol{I}\left(\mathrm{N}_{\mathrm{R}}\right)+\left(\boldsymbol{T}^{-1} \boldsymbol{H}_{\mathrm{c}} \boldsymbol{F}\right)\left(\boldsymbol{T}^{-1} \boldsymbol{H}_{\mathrm{c}} \boldsymbol{F}\right)^{\mathrm{H}}\right|
$$

Equation (34) and (35) indicates that the two transmissions are effective.

\section{OSINR Computation for MMSE}

Consider transmission as

$$
\boldsymbol{y}=\boldsymbol{H}_{\mathrm{e}} \boldsymbol{x}+\boldsymbol{n}_{\mathrm{e}} ; \mathrm{E}\left\{\boldsymbol{x} \boldsymbol{x}^{\mathrm{H}}\right\}=\boldsymbol{I}\left(\mathrm{N}_{\mathrm{S}}\right) ; \mathrm{E}\left\{\boldsymbol{n}_{\mathrm{e}} \boldsymbol{n}_{\mathrm{e}}{ }^{\mathrm{H}}\right\}=\sigma_{\mathrm{e}}{ }^{2} \boldsymbol{I}\left(\mathrm{N}_{\mathrm{R}}\right)
$$

Let $\boldsymbol{x}_{\mathrm{o}}=\boldsymbol{M y}=\boldsymbol{M}\left(\boldsymbol{H}_{\mathrm{e}} \boldsymbol{x}+\boldsymbol{n}_{\mathrm{e}}\right)$, where

$$
\boldsymbol{M}=\underset{M}{\arg \min } \mathrm{E}\left\{\left\|x_{\mathrm{o}}-x\right\|_{\mathrm{F}}^{2}\right\}
$$

According to orthogonality principle,

$$
\begin{gathered}
\mathrm{E}\left\{\left(\boldsymbol{x}_{\mathrm{o}}-\boldsymbol{x}\right) \boldsymbol{y}^{\mathrm{H}}\right\}=\boldsymbol{0} \\
\text { So, } \boldsymbol{M}=\boldsymbol{H}_{\mathrm{e}}{ }^{\mathrm{H}}\left(\boldsymbol{H}_{\mathrm{e}} \boldsymbol{H}_{\mathrm{e}}{ }^{\mathrm{H}}+\sigma_{\mathrm{e}}{ }^{2} \boldsymbol{I}\right)^{-1}
\end{gathered}
$$

Let, $\boldsymbol{D}=\operatorname{diag}\left(\boldsymbol{M H}_{\mathrm{e}}\right), \boldsymbol{N}=\operatorname{diag}\left(\sigma_{\mathrm{e}}^{2} \boldsymbol{M} \boldsymbol{M}^{\mathrm{H}}\right)$ and $\boldsymbol{I}_{\mathrm{f}}=$ $\boldsymbol{M H}_{\mathrm{e}}-\boldsymbol{D}$, then OSINR for each symbol in the transmitting signal vector is

$$
\gamma_{i}=\left(\boldsymbol{D} \boldsymbol{D}^{\mathrm{H}}\right)_{i i} /\left[\left(\boldsymbol{I}_{\mathrm{f}} \boldsymbol{I}_{\mathrm{f}}{ }^{\mathrm{H}}\right)_{i i}+(\boldsymbol{N})_{i i}\right] ; i=1,2, \ldots, \mathrm{N}_{\mathrm{S}}
$$

$(\boldsymbol{A})_{i i}$ means the $i^{\text {th }}$ row and $i^{\text {th }}$ column element of ma$\operatorname{trix} \boldsymbol{A}$.

\section{Proof of Lemma 1}

According to Equation (20) and (21), BLER is one-one to RBIR. Then consider the uncoded block, there is 


$$
B L E R_{\mathrm{u}}=\operatorname{RBIRtoBLER}\left(R B I R_{\mathrm{u}}\right)
$$

Since the MCS is given, it is pointed out that Extrinsic Information Transfer (EXIT) is definite [11]. So RBIR for the coded block after iterative decoding is determined by

$$
R B I R=\operatorname{EXIT}_{\mathrm{MCS}}\left(R B I R_{\mathrm{u}}\right)
$$

So there is

$$
\begin{aligned}
B L E R & =\text { RBIRtoBLER }(R B I R) \\
& =\operatorname{RBIRtoBLER}\left[\operatorname{EXIT}_{\mathrm{MCS}}\left(R_{B} B R_{\mathrm{u}}\right)\right]
\end{aligned}
$$

$=$ RBIRtoBLER $\left\{\right.$ EXIT $_{\mathrm{MCS}}\left[\right.$ InversRBIRtoBLER $\left.\left.\left(B L E R_{\mathrm{u}}\right)\right]\right\}$

$=$ MappingFunction $_{\mathrm{MCS}}\left(B L E R_{\mathrm{u}}\right)$

This is referred to lemma 1 .

\section{Normalized MI for SISO}

For SISO transmission, the received symbol is

$$
y=H x+n ; \mathrm{E}\left\{x x^{*}\right\}=1 ; \mathrm{E}\left\{n n^{*}\right\}=\sigma^{2}=10^{-S N R / 10}
$$

$I$ is computed as

$$
I=\frac{1}{\log _{2} \mathrm{~N}_{\mathrm{QAM}}} \underset{x, y}{\mathrm{E}}\left\{\log _{2} \frac{P(y \mid x)}{P(y)}\right\}=\frac{M I}{\log _{2} \mathrm{~N}_{\mathrm{QAM}}}
$$

Since $x$ is random selected from the constellation, then

$$
P\left(x=q_{i}\right)=1 / \mathrm{N}_{\mathrm{QAM}}
$$

Where $q_{i}$ is the $i^{\text {th }}$ mapping point in the modulation constellation, and NQAM is the number of points in the constellation. So

$$
M I=\frac{\sum_{i=1}^{\mathrm{N}_{\mathrm{OAM}}} \mathrm{E}_{y}\left\{\log _{2} \mathrm{~N}_{\mathrm{QAM}} P\left(y \mid q_{i}\right) / \sum_{i=1}^{\mathrm{N}_{\mathrm{OAM}}} P\left(y \mid q_{k}\right)\right\}}{\mathrm{N}_{\mathrm{QAM}}}
$$

Then consider the probability of $P(y \mid x)$,

$$
\begin{aligned}
& P(y \mid x)=P(n=y-H x) \\
& =P\left(n_{\text {real }}=(y-H x)_{\text {real }}\right) P\left(n_{\text {imag }}=(y-H x)_{\text {imag }}\right) \\
& =\frac{1}{\sqrt{\pi} \sigma} \exp \left(-\frac{(y-H x)_{\text {real }}^{2}}{\sigma^{2}}\right) \frac{1}{\sqrt{\pi} \sigma} \exp \left(\frac{(y-H x)_{\text {imag }}^{2}}{\sigma^{2}}\right) \\
& =\frac{1}{\sqrt{\pi} \sigma} \exp \left(-\frac{|y-H x|^{2}}{\sigma^{2}}\right)
\end{aligned}
$$

Let $\Delta_{i, k}=q_{i}-q_{k}$,

$$
M I=\frac{\sum_{i=1}^{\mathrm{N}_{\mathrm{QAM}}} \oint_{n} p(n) \log _{2} \frac{\mathrm{N}_{\mathrm{QAM}} \exp \left(-\frac{|n|^{2}}{\sigma^{2}}\right)}{\sum_{i=1}^{\mathrm{N}_{\mathrm{OAM}}} \exp \left(-\frac{\left|H \Delta_{i, k}+n\right|^{2}}{\sigma^{2}}\right)} d n}{\mathrm{~N}_{\mathrm{QAM}}}
$$

Here

$$
p(n)=\exp \left(-|n|^{2} / \sigma^{2}\right) / \pi \sigma^{2}
$$

Let $n_{\mathrm{e}}=n / H$, and $\sigma_{\mathrm{e}}^{2}=\sigma^{2} /|H|^{2}$ then

$$
p\left(n_{\mathrm{e}}\right)=\exp \left(-\left|n_{\mathrm{e}}\right|^{2} / \sigma_{\mathrm{e}}^{2}\right) / \pi \sigma_{\mathrm{e}}^{2}
$$

So

$$
\begin{aligned}
& \sum_{i=1}^{\mathrm{N}_{\mathrm{QAM}}} \oint_{n} p\left(n_{\mathrm{e}}\right) \log _{2} \frac{\mathrm{N}_{\mathrm{QAM}} \exp \left(-\frac{\left|n_{\mathrm{e}}\right|^{2}}{\sigma_{\mathrm{e}}{ }^{2}}\right)}{\sum_{i=1}^{\mathrm{N}_{\mathrm{QAM}}} \exp \left(-\frac{\left|H \Delta_{i, k}+n_{\mathrm{e}}\right|^{2}}{\sigma^{2}}\right)} d n_{\mathrm{e}} \\
& =\frac{\mathrm{N}_{\mathrm{QAM}}}{\mathrm{SISO}_{-} \mathrm{MI}\left(10^{-S N R / 10} /|H|^{2}\right)}
\end{aligned}
$$

So

$$
I=\frac{1}{\log _{2} \mathrm{~N}_{\mathrm{QAM}}} \mathrm{SISO}_{-} \operatorname{MI}\left(10^{-S N R / 10} /|H|^{2}\right)
$$

\section{Normalized MI for $2 \times 2$ MIMO}

$2 \times 2$ MIMO received symbol is

$$
y=\left[\begin{array}{l}
y_{1} \\
y_{2}
\end{array}\right]=\boldsymbol{H} \boldsymbol{x}+\boldsymbol{n}=\left[\begin{array}{ll}
h_{11} & h_{12} \\
h_{21} & h_{22}
\end{array}\right]\left[\begin{array}{l}
x_{1} \\
x_{2}
\end{array}\right]+\left[\begin{array}{l}
n_{1} \\
n_{2}
\end{array}\right]
$$

Where

$$
\left\{\begin{array}{c}
\mathrm{E}\left\{x_{1} x_{1}^{*}\right\}=\mathrm{E}\left\{x_{2} x_{2}^{*}\right\}=1 \\
\mathrm{E}\left\{n_{1} n_{1}^{*}\right\}=\mathrm{E}\left\{n_{2} n_{2}^{*}\right\}=\sigma /^{2}=10^{-S N R / 10} \\
\mathrm{E}\left\{x_{1} x_{2}^{*}\right\}=0 \quad \mathrm{E}\left\{n_{1} n_{2}^{*}\right\}=0
\end{array}\right.
$$

For example, the normalized MI of $x_{1}$ is

$$
I_{1}=\frac{1}{\log _{2} \mathrm{~N}_{\mathrm{QAM}}} \underset{x, y}{\mathrm{E}}\left\{\log _{2} \frac{P\left(x_{1}, \boldsymbol{y} \mid \boldsymbol{x}\right)}{P(\boldsymbol{y})}\right\}
$$

Since $x_{1}$ and $x_{2}$ are random selected from the constel- 
lation,

$$
P\left(x_{1}=q_{1, i}, x_{2}=q_{2, j}\right)=1 / \mathrm{N}_{\mathrm{QAM}}{ }^{2}
$$

Here $q_{1, i}$ and $q_{2, j}$ are the $i^{\text {th }}$ and $j^{\text {th }}$ mapping points in the constellation for $x_{1}$ and $x_{2}$ respectively. $\mathrm{N}_{\mathrm{QAM}}$ is the number of points in the constellation. Given transmitting vector,

$\boldsymbol{q}_{l}=\left[q_{1, i,}, q_{2, j}\right]^{\mathrm{T}} ; l=1,2, \ldots, \mathrm{N}_{\mathrm{QAM}}{ }^{2} ; i, j=1,2, \ldots, \mathrm{N}_{\mathrm{QAM}}$

$$
\text { Let } \boldsymbol{\Delta}_{l, m}=\boldsymbol{H}\left(\boldsymbol{q}_{l}-\boldsymbol{q}_{m}\right) \text {, }
$$

$$
\begin{gathered}
P(y)=\frac{\sum_{m=1}^{\mathrm{N}_{\mathrm{QAM}}{ }^{2}} \exp \left(-\left\|H\left(\boldsymbol{q}_{1}-\boldsymbol{q}_{m}\right)+\boldsymbol{n}\right\|_{\mathrm{F}}^{2} / \sigma^{2}\right)}{\mathrm{N}_{\mathrm{QAM}}{ }^{2} \pi^{2} \sigma^{2}} \\
=\frac{\sum_{m=1}^{\mathrm{N}_{\mathrm{OAM}}{ }^{2}} \exp \left(-\left\|\Delta_{l, m}+\boldsymbol{n}\right\|_{\mathrm{F}}^{2} / \sigma^{2}\right)}{\mathrm{N}_{\mathrm{QAM}}{ }^{2} \pi^{2} \sigma^{4}} \\
P\left(q_{1, i}, \boldsymbol{y} \mid \boldsymbol{q}_{l}\right)=\frac{\sum_{t=1}^{\mathrm{N}_{\mathrm{QAM}}} \exp \left(-\left\|\boldsymbol{H}\left[\begin{array}{c}
q_{1, i}-q_{1, i} \\
q_{2, j}-q_{2, t}
\end{array}\right]+\left[\begin{array}{l}
n_{1} \\
n_{2}
\end{array}\right]\right\|_{\mathrm{F}}^{2} / \sigma^{2}\right)}{\mathrm{N}_{\mathrm{QAM}} \pi^{2} \boldsymbol{\sigma}^{4}} \\
=\frac{\sum_{t=1}^{\mathrm{N}_{\mathrm{QAM}}} \exp \left(-\left\|H\left[\begin{array}{c}
0 \\
q_{2, j}-q_{2, t}
\end{array}\right]+\left[\begin{array}{l}
n_{1} \\
n_{2}
\end{array}\right]\right\|_{\mathrm{F}}^{2} / \sigma^{2}\right)}{\mathrm{N}_{\mathrm{QAM}} \pi^{2} \sigma^{4}}
\end{gathered}
$$

Then

$$
\begin{gathered}
I_{1}=\frac{\sum_{l=1}^{\mathrm{N}_{\mathrm{OAM}}{ }^{2}} \oint_{n} p(\boldsymbol{n}) \log _{2} \frac{p\left(q_{1, i}, \boldsymbol{y} \mid \boldsymbol{q}_{l}\right)}{p(\boldsymbol{y})} d \boldsymbol{n}}{\mathrm{N}_{\mathrm{QAM}}{ }^{2} \log _{2} \mathrm{~N}_{\mathrm{QAM}}} \\
=\frac{\sum_{l=1}^{\mathrm{N}_{\mathrm{OAM}}{ }^{2}} \oint_{n} p(\boldsymbol{n}) \log _{2} \frac{p\left(q_{1, i}, \boldsymbol{y} \mid \boldsymbol{q}_{l}\right) / p(\boldsymbol{n})}{p(\boldsymbol{y}) / p(\boldsymbol{n})} d \boldsymbol{n}}{\mathrm{N}_{\mathrm{QAM}^{2}} \log _{2} \mathrm{~N}_{\mathrm{QAM}}} \\
=\frac{\frac{1}{\mathrm{~N}_{\mathrm{QAM}}{ }^{2}} \sum_{l=1}^{\mathrm{N}_{\mathrm{OAM}}{ }^{2}} \oint_{n} p(\boldsymbol{n}) \log _{2} \frac{p(\boldsymbol{n})}{p(\boldsymbol{y})} d \boldsymbol{n}}{\log _{2} \mathrm{~N}_{\mathrm{QAM}}}
\end{gathered}
$$

$$
\begin{aligned}
& =\frac{\frac{1}{\mathrm{~N}_{\mathrm{QAM}}{ }^{2}} \sum_{l=1}^{\mathrm{N}_{\mathrm{OAM}}{ }^{2}} \oint_{n} p(\boldsymbol{n}) \log _{2} \frac{p(\boldsymbol{n})}{p\left(q_{1, i}, \boldsymbol{y} \mid \boldsymbol{q}_{l}\right)} d \boldsymbol{n}}{\log _{2} \mathrm{~N}_{\mathrm{QAM}}} \\
& =\frac{1}{\log _{2} \mathrm{~N}_{\mathrm{QAM}}}\left(M I_{c}-M I_{2}\right)
\end{aligned}
$$

Consider $M I_{\mathrm{c}}$,

$$
\begin{array}{r}
M I_{\mathrm{c}}=\frac{1}{\mathrm{~N}_{\mathrm{QAM}}{ }^{2}} \sum_{l=1}^{\mathrm{N}_{\mathrm{OAM}}{ }^{2}} \oint_{n} p(\boldsymbol{n}) \log _{2} \frac{p(\boldsymbol{n})}{p(\boldsymbol{y})} d \boldsymbol{n} \\
\frac{\sum_{l=1}^{\mathrm{N}_{\mathrm{OAM}}{ }^{2}} \oint_{n} p(\boldsymbol{n}) \log _{2} \frac{\mathrm{N}_{\mathrm{QAM}^{2}}{ }^{2} \exp \left(-\|\boldsymbol{n}\|_{\mathrm{F}}^{2} / \sigma^{2}\right)}{\sum_{m=1}^{\mathrm{N}_{\mathrm{OAM}}} \exp \left(-\left\|\Delta_{l, m}+\boldsymbol{n}\right\|_{\mathrm{F}}^{2} / \sigma^{2}\right)}}{\mathrm{N}_{\mathrm{QAM}}{ }^{2}} d \boldsymbol{n}
\end{array}
$$$$
\frac{\sum_{l=1}^{\mathrm{N}_{\mathrm{QAM}}{ }^{2}} \oint_{n} p(\boldsymbol{n}) \log _{2} \frac{\mathrm{N}_{\mathrm{QAM}}{ }^{2}}{\sum_{m=1}^{\mathrm{N}_{\mathrm{OAM}}{ }^{2}} \exp \left(-\frac{\left\|\Delta_{l, m}\right\|_{\mathrm{F}}{ }^{2}+\Delta_{l, m}{ }^{H} \boldsymbol{n}+\boldsymbol{n}^{H} \Delta_{l, m}}{\sigma^{2}}\right)} d \boldsymbol{n}}{\mathrm{N}_{\mathrm{QAM}}{ }^{2}}
$$

Let $n_{\mathrm{e}}=\left(\boldsymbol{\Delta}_{l, m}{ }^{\mathrm{H}} \boldsymbol{n}+\boldsymbol{n}^{\mathrm{H}} \boldsymbol{\Delta}_{l, m}\right) /\left\|\boldsymbol{\Delta}_{l, m}\right\|_{\mathrm{F}}$, so

$$
n_{\mathrm{e}} \sim \mathrm{N}\left(0,2 \sigma^{2}\right)
$$

Then

$$
=\frac{\sum_{l=1}^{\mathrm{N}_{\mathrm{QAM}}{ }^{2}} \oint_{n} p\left(n_{\mathrm{e}}\right) \log _{2} \frac{\mathrm{N}_{Q \mathrm{AM}}{ }^{2}}{\sum_{m=1}^{\mathrm{N}_{\mathrm{QAM}}^{2}} \exp \left(-\frac{\left\|\Delta_{l, m}\right\|_{\mathrm{F}}^{2}+\left\|\Delta_{l, m}\right\|_{\mathrm{F}} n_{e}}{\sigma^{2}}\right)} d n}{\mathrm{~N}_{\mathrm{QAM}}^{2}}
$$

Then define approximation as

$$
\Delta_{l}=\sqrt{-\sigma^{2} \log _{\mathrm{e}}\left(\frac{1}{\mathrm{~N}_{\mathrm{QAM}}{ }^{2}} \sum_{m=1}^{\mathrm{N}_{\mathrm{QAM}}{ }^{2}} \exp \left(-\frac{1}{\beta} \frac{\left\|\Delta_{l, m}\right\|_{\mathrm{F}}^{2}}{\sigma^{2}}\right)\right)}
$$

Here the tuning parameter is

Table 8. Tuning parameter for $2 \times 2$ MIC

\begin{tabular}{lllllll}
\hline \hline $\mathrm{SNR} / \mathrm{dB}$ & {$\left[\begin{array}{llllll}-8 & -5 & -2 & 1 & 4 & 7\end{array}\right]$} \\
\hline$\beta$ & {$\left[\begin{array}{llllll}2 & 2.1 & 2.2 & 2.3 & 2.4 & 2.55\end{array}\right]$}
\end{tabular}


Then

$M I_{c}=\frac{\log _{2} \mathrm{e}}{\sigma^{2} \mathrm{~N}_{\mathrm{QAM}}{ }^{2}} \sum_{l=1}^{\mathrm{N}_{\mathrm{QAM}}^{2}} \Delta_{l}^{2}=\operatorname{VectorMI}(\boldsymbol{H}, S N R)$

(65)

Then compute $M I_{2}$, and let $\Delta_{2, j, t}=q_{2, j}-q_{2, t}$,

$$
\begin{gathered}
M I_{2}=\frac{1}{\mathrm{~N}_{\mathrm{QAM}}{ }^{2}} \sum_{i=1}^{\mathrm{N}_{\mathrm{QAM}}} \sum_{j=1}^{\mathrm{N}_{\mathrm{QAM}}} \oint_{n} p(\boldsymbol{n}) \log _{2} \frac{p(\boldsymbol{n})}{P\left(x_{1}, \boldsymbol{y} \mid \boldsymbol{x}\right)} d \boldsymbol{n} \\
=\frac{1}{\mathrm{~N}_{\mathrm{QAM}}} \sum_{j=1}^{\mathrm{N}_{\mathrm{QAM}}} \oint_{n} p(\boldsymbol{n}) \log _{2} \frac{\exp \left(-\frac{\left|n_{1}\right|^{2}+\left|n_{2}\right|^{2}}{\sigma^{2}}\right)}{\left|h_{12} \Delta_{2, j, t}+n_{1}\right|^{2}} d \boldsymbol{n} \\
\sum_{t=1}^{\mathrm{N}_{\mathrm{QAM}}} \exp \left(-\frac{\left|h_{22} \Delta_{2, j, t}+n_{2}\right|^{2}}{\sigma^{2}}\right)
\end{gathered}
$$

Here

$$
\begin{aligned}
& \left(\left|h_{12} \Delta_{2, j, t}+n_{1}\right|^{2}+\left|h_{22} \Delta_{2, j, t}+n_{2}\right|^{2}\right) /\left(\left|h_{12}\right|^{2}+\left|h_{22}\right|^{2}\right) \\
= & \left|\Delta_{2, j, t}\right|^{2}+\frac{\left|n_{1}\right|^{2}+\left|n_{2}\right|^{2}}{\left|h_{12}\right|^{2}+\left|h_{22}\right|^{2}}+2\left\{\Delta_{2, j, t} \frac{h_{12} n_{1}^{*}+h_{22} n_{2}^{*}}{\left|h_{12}\right|^{2}+\left|h_{22}\right|^{2}}\right\}_{\text {real }}
\end{aligned}
$$

Let $n_{\mathrm{e}}=\left(h_{12} n_{1}{ }^{*}+h_{22} n_{2}{ }^{*}\right) /\left(\left|h_{12}\right|^{2}+\left|h_{22}\right|^{2}\right)$, so $\mathrm{E}\left\{n_{\mathrm{e}}\right\}=0 ; \mathrm{E}\left\{n_{\mathrm{e}, \text { real }} n_{\mathrm{e}, \text { imag }}\right\}=0 ; \mathrm{E}\left\{\left|n_{\mathrm{e}}\right|^{2}\right\}=\sigma_{\mathrm{e}}{ }^{2}$;

$$
\sigma_{\mathrm{e}}^{2}=\sigma^{2} /\left(\left|h_{12}\right|^{2}+\left|h_{22}\right|^{2}\right)
$$

Then

$$
\begin{gathered}
T_{2}=\frac{1}{\mathrm{~N}_{\mathrm{QAM}}} \sum_{j=1}^{\mathrm{N}_{\mathrm{QAM}}} \oint_{n_{\mathrm{e}}} p\left(n_{\mathrm{e}}\right) \log _{2} \frac{\exp \left(-\frac{\left|n_{\mathrm{e}}\right|^{2}}{\sigma_{\mathrm{e}}{ }^{2}}\right)}{\sum_{t=1}^{\mathrm{N}_{\mathrm{QAM}}} \exp \left(-\frac{\left|\Delta_{2, j, t}+n_{\mathrm{e}}\right|^{2}}{\sigma_{\mathrm{e}}{ }^{2}}\right)} d n_{\mathrm{e}} \\
\left.T_{2}=\frac{1}{\mathrm{~N}_{\mathrm{QAM}}} \sum_{j=1}^{\mathrm{N}_{\mathrm{QAM}}} \oint_{n_{\mathrm{e}}} p\left(n_{\mathrm{e}}\right) \log _{2} \frac{\exp \left(-\frac{\left|n_{\mathrm{e}}\right|^{2}}{\sigma_{\mathrm{e}}{ }^{2}} \sum_{t=1}^{\mathrm{N}_{\mathrm{QAM}}} \exp \left(-\frac{\left|\Delta_{2, j, t}+n_{\mathrm{e}}\right|^{2}}{\sigma_{\mathrm{e}}{ }^{2}}\right)\right.}{\sum_{\mathrm{e}}}\right) \\
=\mathrm{SISO}_{-} \mathrm{MI}\left(\frac{10^{-S N R / 10}}{\left|h_{12}\right|^{2}+\left|h_{22}\right|^{2}}\right)
\end{gathered}
$$

The computation of $I_{1}$ and $I_{2}$ are similar, so

$$
\begin{aligned}
& I_{1}= \operatorname{VectorMI}(\boldsymbol{H}, S N R)-\mathrm{SISO}_{-} \mathrm{MI}\left(\frac{10^{-S N R / 10}}{\left|h_{12}\right|^{2}+\left|h_{22}\right|^{2}}\right) \\
& \log _{2} \mathrm{~N}_{\mathrm{QAM}} \operatorname{VectorMI}(\boldsymbol{H}, S N R)-\mathrm{SISO}_{-} \mathrm{MI}\left(\frac{10^{-S N R / 10}}{\left|h_{11}\right|^{2}+\left|h_{21}\right|^{2}}\right) \\
& I_{2}=\frac{\log _{2} \mathrm{~N}_{\mathrm{QAM}}}{}
\end{aligned}
$$

